\title{
ROTATING GLOBULAR CLUSTERS
}

\author{
P. BIANCHINI ${ }^{1}$, A. L. VARRI ${ }^{2}$, G. Bertin, AND A. ZOCCHI \\ Dipartimento di Fisica, Università degli Studi di Milano, via Celoria 16, I-20133 Milano, Italy; bianchini@mpia.de \\ Received 2013 February 16; accepted 2013 May 24; published 2013 July 8
}

\begin{abstract}
Internal rotation is thought to play a major role in the dynamics of some globular clusters. However, in only a few cases has internal rotation been studied by the quantitative application of realistic and physically justified global models. Here, we present a dynamical analysis of the photometry and three-dimensional kinematics of $\omega$ Cen, 47 Tuc, and M15, by means of a recently introduced family of self-consistent axisymmetric rotating models. The three clusters, characterized by different relaxation conditions, show evidence of differential rotation and deviations from sphericity. The combination of line-of-sight velocities and proper motions allows us to determine their internal dynamics, predict their morphology, and estimate their dynamical distance. The well-relaxed cluster 47 Tuc is interpreted very well by our model; internal rotation is found to explain the observed morphology. For M15, we provide a global model in good agreement with the data, including the central behavior of the rotation profile and the shape of the ellipticity profile. For the partially relaxed cluster $\omega$ Cen, the selected model reproduces the complex three-dimensional kinematics; in particular, the observed anisotropy profile, characterized by a transition from isotropy to weakly radial anisotropy and then to tangential anisotropy in the outer parts. The discrepancy found for the steep central gradient in the observed line-of-sight velocity dispersion profile and for the ellipticity profile is ascribed to the condition of only partial relaxation of this cluster and the interplay between rotation and radial anisotropy.
\end{abstract}

Key words: globular clusters: general - globular clusters: individual: NGC 104 (47 Tuc), NGC 5139 ( $\omega$ Cen), NGC 7078 (M15)

\section{INTRODUCTION}

Globular clusters (GCs) have long been considered simple quasi-relaxed nonrotating stellar systems, characterized by spherical symmetry and isotropy in velocity space. Spherical isotropic models (in particular, the King 1966 models and a spherical, nonrotating version of the Wilson 1975 models) have indeed been shown to provide a satisfactory zeroth-order description of the main observed dynamical properties (for a recent dynamical study of a large sample of GCs based on modeling of only the observed photometric profiles, see McLaughlin \& van der Marel 2005, hereafter denoted as MLvdM05; for a dynamical study of a sample of $13 \mathrm{GCs}$ based on both photometric and (line-of-sight) kinematic profiles, see Zocchi et al. 2012, hereafter ZBV12).

However, the acquisition of high-quality data is rapidly bringing us well beyond such a simple picture. In particular, deviations from sphericity have been observed (see Geyer et al. 1983, White \& Shawl 1987, and Chen \& Chen 2010; the last two papers will be denoted as WS87 and CC10, respectively). In addition, significant internal rotation has been detected in a growing number of Galactic GCs from line-ofsight velocity measurements (for a summary, see Table 7.2 in Meylan \& Heggie 1997; for more recent investigations, see, among others, Lane et al. 2011; Bellazzini et al. 2012) and, in a few cases, from kinematical measurements in the plane of the sky (e.g., for M22 see Peterson \& Cudworth 1994; for $\omega$ Cen see van Leeuwen \& Le Poole 2002; and for 47 Tuc see Anderson \& King 2003). Detailed three-dimensional kinematics are therefore available for selected Galactic clusters. As for the

\footnotetext{
1 Now at Max Planck Institute for Astronomy, Königstuhl 17, D-69117 Heidelberg, Germany.

2 Now at Department of Astronomy, Indiana University, 727 East 3rd Street, Swain West 319, Bloomington, IN 47405-7105, USA.
}

measurement of proper motions, the Hubble Space Telescope (HST) is best used to probe the central regions of the systems (McLaughlin et al. 2006; Anderson \& van der Marel 2010), whereas ground-based observations are considered for widefield coverage (van Leeuwen et al. 2000; Bellini et al. 2009; Sariya et al. 2012; Zloczewski et al. 2012). The future mission Gaia is planned to provide three-dimensional kinematical data for a large number of stars in GCs (except for the center of very dense objects affected by crowding). All this progress calls for the development of a more complete and realistic dynamical modeling framework, in which internal rotation and deviations from sphericity are fully taken into consideration.

Internal rotation, external tides, and pressure anisotropy are the main physical factors that could be responsible for the observed flattening of GCs, but we still do not know which is the dominant cause of the observed deviations from spherical symmetry (van den Bergh 2008). In this paper, we will not address the effect of tides because they are expected to act mainly in the outer parts of these stellar systems, in regions outside the focus of the present investigation. The suggestion that internal rotation plays a role in determining the structure and morphology of GCs is not new (King 1961; Fall \& Frenk 1985). A tool commonly used to determine the importance of rotation in shaping a stellar system is the $V / \sigma$ versus $\varepsilon$ diagram (Davies et al. 1983; Binney 2005; Cappellari et al. 2007). Given their small ellipticities $(\varepsilon<0.35)$, GCs are typically located in the portion of the diagram representing configurations characterized by solid-body rotation and isotropy to mild anisotropy of the velocity dispersion tensor. However, this approach provides only a zeroth-order description of the dynamical interplay between internal rotation and pressure anisotropy. In particular, such a diagram considers only global quantities, which can vary significantly as a result of detailed changes with radius of the anisotropy parameter and of inclination effects. These factors are 
Table 1

Properties of $\omega$ Cen, 47 Tuc, and M15

\begin{tabular}{|c|c|c|c|c|c|c|c|c|c|c|c|}
\hline GC & $\begin{array}{c}d \\
(1)\end{array}$ & $\begin{array}{l}R_{\mathrm{c}} \\
(2)\end{array}$ & $\begin{array}{c}C \\
(3)\end{array}$ & $\begin{array}{c}\log T_{\mathrm{c}} \\
\quad(4)\end{array}$ & (5) & (6) & $\begin{array}{c}\phi \\
(7)\end{array}$ & $\begin{array}{c}\text { P.A. } \\
\text { (8) }\end{array}$ & $\begin{array}{c}i \\
(9)\end{array}$ & $\begin{array}{l}N_{\operatorname{los}} \\
(10)\end{array}$ & $\begin{array}{l}N_{\mathrm{pm}} \\
(11)\end{array}$ \\
\hline$\omega$ Cen & $5.2 \pm 0.7$ & $142.20 \pm 8.26$ & $1.31 \pm 0.04$ & $9.52 \pm 0.04$ & $0.21 \pm 0.02$ & $0.17 \pm 0.00$ & $6 \pm 0$ & $12 \pm 1$ & $50 \pm 4$ & 1868 & $2740+72970$ \\
\hline M15 & $10.4 \pm 0.8$ & $8.40 \pm 0.95$ & $2.29 \pm 0.18$ & $7.62 \pm 0.06$ & $0.19 \pm 0.10$ & $0.05 \pm 0.00$ & $215 \pm 1$ & $106 \pm 1$ & $60 \pm 15$ & 1777 & 703 \\
\hline
\end{tabular}

Notes. For each cluster we list: (1) the distance from the Sun $d$ in kpc; (2) the core radius $R_{\mathrm{c}}$ in arcsec; (3) the concentration parameter $C$; (4) the logarithm of the core relaxation time $T_{\mathrm{c}}$ in years from spherical King models; the ellipticity $\varepsilon=1-b_{p} / a_{p}$ (where $a_{p}$ and $b_{p}$ indicate the observed major and minor axes, respectively), as reported by (5) CC10 and (6) WS87; (7) the position angle (P.A.) of the photometric minor axis $\phi$ measured in degrees (east of north); (8) the position angle of the kinematic rotation axis P.A. on the plane of the sky measured in degrees (east of north); (9) the inclination $i$ of the rotation axis with respect to the line of sight measured in degrees; the number of data points for the samples of (10) line-of-sight velocities $N_{\text {los }}$; and (11) proper motions $N_{\mathrm{pm}}$.

References. From Columns 1-3: Harris 2010; Column 4: ZBV12; Column 5: CC10; Columns 6 and 7: WS87; Column 9: van de Ven et al. (2006), Anderson \& King (2003), van den Bosch et al. (2006) (from top to bottom, that is, for $\omega$ Cen, 47 Tuc, and M15, respectively); Columns 8, 10, and 11: considered in the present work.

generally not well constrained observationally (see Section 7.3 and Figure 14). Therefore, the present investigation is motivated by the need to provide a more realistic dynamical interpretation of selected rotating Galactic GCs, with particular attention paid to objects that show small yet significant deviations from the behavior of a simple isotropic (slow) rotator. In this respect, the most significant investigations made so far are the orbitbased axisymmetric modeling of $\omega$ Cen and M15 (van de Ven et al. 2006; van den Bosch et al. 2006, respectively), the study of $\omega$ Cen by means of axisymmetric Wilson (1975) models (Sollima et al. 2009) and an oblate rotator nonparametric model (Merritt et al. 1997), the description of M13 by means of a family of models with three integrals of motion (Lupton \& Gunn 1987; Lupton et al. 1987), and the analysis of the internal dynamics of a small sample of Galactic GCs through dedicated two-dimensional Fokker-Planck models (Fiestas et al. 2006).

Additional interest in the role of rotation derives from the fact that the presence of global angular momentum is expected to change the long-term dynamical evolution of stellar systems with respect to the traditional paradigm based on nonrotating models (for a summary, see Heggie \& Hut 2003). Numerical investigations, primarily based on a Fokker-Planck approach (Einsel \& Spurzem 1999; Kim et al. 2002, 2008; Hong et al. 2013), demonstrate that, in general, the presence of rotation accelerates dynamical evolution.

Internal rotation may also play an indirect role in the controversial issue of the presence of intermediate-mass black holes (IMBHs) in GCs. In fact, sizable central gradients in the velocity dispersion profiles are often ascribed to the presence of an IMBH (Baumgardt et al. 2005). A critical discussion of the observed gradients is often reduced to the application of the Jeans equations in which variations of the slope of the velocity dispersion profile are obtained by varying only the amount of pressure anisotropy (without considering rotation; e.g., see Lanzoni et al. 2013; Lützgendorf et al. 2011; van der Marel $\&$ Anderson 2010). However, differential rotation and pressure anisotropy can cooperate to produce nontrivial gradients in the velocity dispersion profiles (see Varri \& Bertin 2012, hereafter denoted as VB12) and might thus be important elements to consider in the interpretation of the data.

In view of these motivations, a new family of self-consistent axisymmetric models has been introduced recently, specifically designed to describe quasi-relaxed stellar systems with finiteglobal angular momentum (VB12); the models are characterized by differential rotation, approximately rigid in the center and vanishing in the outer parts, and pressure anisotropy.
In the present paper, we apply this family of differentially rotating global models to three Galactic GCs, namely, $\omega$ Cen, 47 Tuc, and M15, that have been observed in detail and are known to exhibit evidence for rotation. In Section 2, we present the available data sets for these three GCs and describe the procedure followed to construct the profiles of the relevant photometric and kinematic quantities (some important detailed descriptions are provided separately in Appendices A and B). In Section 3, we summarize the property of the adopted family of self-consistent rotating dynamical models and introduce the method used to identify the best model to describe the data available for the three clusters. The detailed results on $\omega$ Cen, 47 Tuc, and M15 are reported in Sections 4-6, taking into consideration that $\omega$ Cen is only partially relaxed, while 47 Tuc and M15 are fully relaxed. In Section 7, we discuss the results of the present paper and compare them with those obtained from previous studies. Finally, in Section 8, we summarize the conclusions that can be drawn from our study.

\section{OBSERVED KINEMATIC AND PHOTOMETRIC PROFILES}

In this section, we describe the kinematic and photometric data sets that we will use in the dynamical analysis and the methods we will use to build the relevant profiles. We pay particular attention to the construction of the rotation profiles. Table 1 summarizes the basic properties of the GCs $\omega$ Cen, 47 Tuc, and M15.

\subsection{Kinematic Profiles}

We gathered and combined kinematic data sets covering a large radial extent from the literature. In Appendix A, we describe in detail the data sets selected for the line-of-sight velocities and proper motions. The data are referenced to a Cartesian coordinate system $\left(x_{p}, y_{p}\right)$, with $x_{p}$ and $y_{p}$ aligned with the major and minor axes, respectively (van de Ven et al. 2006). The $z_{p}$-axis identifies the line-of-sight direction. Proper motions are then decomposed into projected tangential $\mu_{t}$ and radial $\mu_{R}$ components.

The present dynamical study is based on a combined analysis of the following kinematic profiles: (1) rotation profiles, (2) velocity dispersion profiles, and (3) pressure anisotropy profiles. The kinematic profiles are constructed with the traditional binning approach; that is, the data are divided into bins containing an equal number of stars. In particular, radial bins are 
used to construct the velocity dispersion and anisotropy profiles, whereas the line-of-sight rotation profile is constructed by binning along the observed major axis, in intervals of $x_{p}$. We choose a number of bins that represents the best compromise between having a rich radial sampling and accurate points, ${ }^{3}$ as in ZBV12.

To calculate the mean velocity and the velocity dispersion, with associated errors, we apply a maximum likelihood technique to the data, following the method described by Pryor \& Meylan (1993) in which non-constant velocity errors are taken into consideration. ${ }^{4}$ The details of the procedure used to obtain the different profiles are given below.

\subsubsection{Rotation Profiles}

The first step in building a rotation profile consists of identifying the position angle (P.A.) of the projected rotation axis in the plane of the sky (defined as the angle between the rotation axis and the north direction, measured east of north). To identify the P.A., the following standard procedure is used (e.g., see Côté et al. 1995; Bellazzini et al. 2012): the line-of-sight velocities data set is divided in two halves by a line passing through the center with a given P.A. and for each subsample the mean lineof-sight velocity is computed. The P.A. is varied in steps of $10^{\circ}$ and the difference between the mean velocities $\Delta V$ is plotted against P.A. The resulting pattern is fit with a sine function (see Figure 1); the P.A. at which the maximum difference in mean velocities is reached corresponds to the rotation axis and the amplitude $A$ of the sine function gives an estimate of the significance of the internal rotation. The values obtained for the P.A. are used to rotate the Cartesian coordinate system in the plane of the sky by aligning $x_{p}$ and $y_{p}$ with the major and minor axes, respectively (Appendix A). The results are listed in Table 1 and are compared to the P.A.s of the photometric minor axes $\phi$ reported by WS87.

The P.A.s of the kinematic minor axes of $\omega$ Cen and 47 Tuc are in reasonable agreement with the photometric ones, suggesting a direct connection between the presence of internal rotation and observed flattening. A discrepancy is found instead for M15: for this cluster, the small observed flattening $(\varepsilon \approx 0.05)$ makes the identification of the minor axis nontrivial. Various estimates of the photometric P.A. are given in the literature, ranging from $215^{\circ}$ to $135^{\circ}$, suggesting a possible twisting of the P.A. of both the photometric and kinematic minor axes (Gebhardt et al. 2000; van den Bosch et al. 2006). Additional tests of the twisting of the rotation axis and of the radial variation of the rotation amplitude are recorded in Appendix B.

After identifying the rotation axis, we can proceed to build the rotation profiles. First, we subtract from each data set the measured mean systemic velocity. Then, we divide the line-ofsight velocities data set in bins along the major axis $x_{p}$; each bin is assigned an average $x$ position, mean velocity, and associated uncertainty. In the case of the proper-motion data set, the rotation profile is constructed by dividing the data set in radial bins and computing for each bin the mean radial distance and the mean velocity, separately for the tangential and projected radial components. We then end up with three mean-velocity profiles,

\footnotetext{
3 The number of stars per bin is chosen to be large enough to limit the uncertainties associated with low-number statistics (for the profiles constructed in this paper, the number of stars per bin is $>90$ ).

4 A contamination model is not included in the maximum likelihood estimator, since potential non-members have already been excluded in the data sets that have been considered.
}
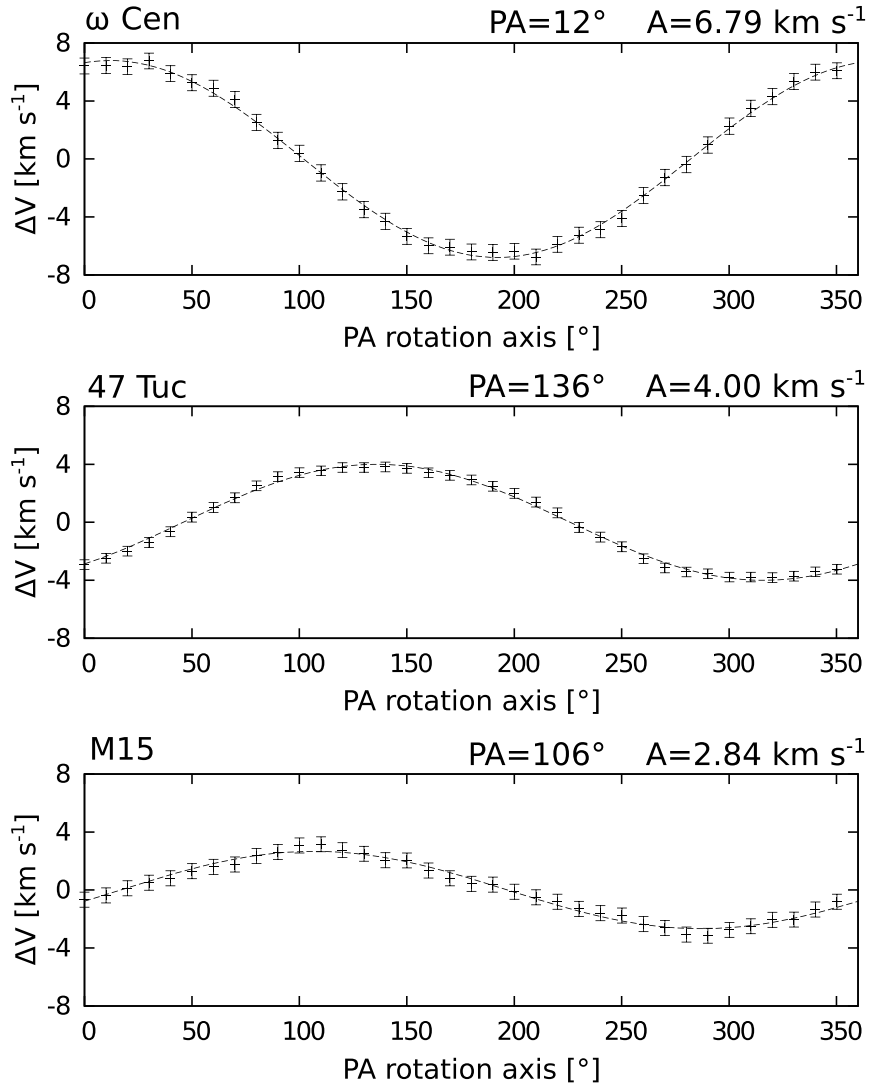

Figure 1. Difference of the mean velocities calculated on each side of the system divided by a line passing through the center with a given position angle, P.A. The P.A. at which the maximum difference is reached corresponds to the position of the rotation axis. The best-fit sine function is plotted (solid line) and the corresponding P.A. and amplitude $A$ are indicated.

one for the line of sight, $V_{\text {los }}\left(x_{p}\right)$, and two for the proper motions, $V_{\mathrm{t}}(R)$ and $V_{\mathrm{R}}(R)$.

\subsubsection{Velocity Dispersion and Anisotropy Profiles}

The velocity dispersion profiles are computed by dividing the data sets into radial bins; we consider the mean velocity of the entire data set as a constant value throughout the cluster, and we calculate the velocity dispersion for each bin with the associated uncertainty. For each bin, the distance from the center is taken to be the mean of the radial positions of the stars that it contains. The profiles obtained are $\sigma_{\text {los }}(R), \sigma_{\mathrm{t}}(R)$, and $\sigma_{\mathrm{R}}(R)$, for the line-of-sight velocities, projected tangential component of proper motions, and projected radial component of proper motions, respectively.

From the dispersion profiles of the proper motions, we also calculate the anisotropy profile. This quantity is defined here as the ratio of the velocity dispersion in the tangential component to the velocity dispersion in the radial component, $\sigma_{\mathrm{t}}(R) / \sigma_{\mathrm{R}}(R)$. Values of $\sigma_{\mathrm{t}} / \sigma_{\mathrm{R}} \approx 1$ indicate isotropy in velocity space, values of $\sigma_{\mathrm{t}} / \sigma_{\mathrm{R}}>1$ indicate the presence of tangential anisotropy, and values of $\sigma_{\mathrm{t}} / \sigma_{\mathrm{R}}<1$ indicate radial anisotropy.

\subsection{Photometric Profiles}

The photometric quantities that we will use in the dynamical analysis are the surface brightness profile and the ellipticity profile. Below, we briefly describe the data sets available for the construction of these profiles. 


\subsubsection{Surface Brightness Profiles}

The surface brightness profiles are taken from ZBV12: they are $V$-band surface brightness profiles built from the data of Trager et al. (1995), divided into circular annuli so that the surface brightness measured in mag $\operatorname{arcsec}^{-2}$ is reported as a function of projected radius. The profiles are extinction corrected, under the assumption of constant extinction over the entire extent of the cluster.

Since the central regions correspond to the least reliable parts of the profiles of Trager et al. (1995), a combination of different data sets is needed. The more accurate data available from Noyola \& Gebhardt (2006) are used for 47 Tuc and M15. For 47 Tuc, the data from the two sources are simply co-added; for M15, the two data sets are combined by removing the points from Trager et al. (1995) that do not agree with the more recent profile. In the case of $\omega$ Cen, the inner points kindly provided by E. Noyola (Noyola et al. 2008) are added to the Trager et al. (1995) surface brightness profile.

\subsubsection{Ellipticity}

From a morphological point of view, GCs present only small deviations from spherical symmetry. Yet there is observational evidence of flattening, as measured by the ellipticity parameter, defined as $\varepsilon=1-b_{p} / a_{p}$, where $b_{p} / a_{p}$ is the ratio of the minor to major axis of the projected image of a cluster in the plane of the sky. For a long time, the WS87 database represented the only comprehensive collection of ellipticity measurements for Galactic GCs; recently, an alternative homogeneous database of ellipticities has been published by $\mathrm{CC} 10$. The two distributions of values show significant differences: in fact, from the WS87 database (93 objects), Galactic clusters appear to be predominantly round, with the peak of the distribution at $\varepsilon \approx 0.05$, maximum value at $\varepsilon \approx 0.3$, and axial ratios randomly oriented in space. In contrast, the distribution of the CC10 ellipticities (116 objects, 82 in common with the other database) is peaked at $\varepsilon \approx 0.15$ with a maximum value of $\varepsilon \approx 0.45$. In addition, especially for the clusters in the region of the Galactic bulge, their major axes point preferentially toward the Galactic center.

The apparent discrepancies between the two studies should be interpreted by taking into account that (1) WS87 ellipticities result from an optical study, with the use of a surface photometry technique based on the blurring of the digitized images of blue-sensitive photographic plates. In turn, CC10 ellipticities are determined with a number-count technique, based on the analysis of the spatial distribution of Two Micron All Sky Survey point sources. (2) Also, as a result of the different resolution limits of the two approaches, WS87 measurements mostly refer to the inner regions of the clusters, whereas CC10 measurements refer to the outer parts. Unfortunately, in both cases, the flattening values do not refer to a standard isophote, such as the cluster half-light radius (see Kontizas et al. 1989). This is an intrinsic limitation, because there is observational evidence that the ellipticity of a cluster depends on radius (see Geyer et al. 1983).

In the present paper, we will use the ellipticity profile of $\omega$ Cen taken from Geyer et al. (1983). It is the most extended ellipticity profile available for a Galactic GC, as it reaches $\approx 0.5 r_{\text {tr }}$, where $r_{\text {tr }}$ represents the standard truncation radius. In addition, Anderson \& van der Marel (2010) report the ellipticity profile of the central region $(R \lesssim 250 \mathrm{arcsec})$; in the following analysis, both data sets will be taken into consideration. For 47 Tuc and M15, we will use the profiles of Figure 5 in WS87. They reach $\approx 0.2 r_{\text {tr }}$ and $\approx 0.4 r_{\text {tr }}$, respectively. We note that a genuine radial variation is present in the three ellipticity profiles. This is particularly evident for $\omega$ Cen, which exhibits nonmonotonic behavior.

\section{MODEL IDENTIFICATION AND PREDICTIONS}

The family of self-consistent axisymmetric models that we will consider has been specifically designed to describe quasirelaxed stellar systems with finite global angular momenta (VB12). These models are global, finite-mass solutions of the self-consistent problem associated with the distribution function $f_{\mathrm{WT}}^{d}(I)$ (see Equation (21) in VB12), in which the integral of the motion $I=I\left(E, J_{z}\right)$ is defined as

$$
I\left(E, J_{z}\right)=E-\frac{\omega J_{z}}{1+b J_{z}^{2 c}},
$$

where $\omega, b$, and $c>1 / 2$ are positive constants. The subscript WT in the distribution function is a reminder that the form of the function is that of the corresponding spherical, isotropic, nonrotating models characterized by Wilson truncation; a full description of the physical arguments that led to this choice of distribution function is provided in VB12. The integral of the motion reduces to $I \sim E$ for high values of $J_{z}$ and to the Jacobi integral $I \sim H=E-\omega J_{z}$ for low values of $J_{z}$. Therefore, the models are characterized by differential rotation, approximately rigid in the center and vanishing in the outer parts. The models are defined by four dimensionless parameters. Two dimensionless parameters are the concentration parameter $\Psi$ (this parameter is often denoted as $W_{0}$ in the description of the King models), defined as the depth of the dimensionless potential well at the center of the cluster, and the rotationstrength parameter $\chi=\omega^{2} /\left(4 \pi G \rho_{0}\right)$. The parameters $b$ (or, equivalently, the dimensionless parameter $\bar{b}$; see definition in VB12) and $c$ determine the shape of the rotation profile. For the purposes of the present study, we checked that varying $c$ does not introduce significant differences; thus, we decided to simplify our investigation by setting $c=1$. The effect of adopting a larger value of $\bar{b}$ produces models in which the solid-body rotation covers a wider radial range. For the self-consistent models, the velocity dispersion tensor is characterized by isotropy in the central region, weak radial anisotropy in the intermediate regions, and tangential anisotropy in the outer parts. ${ }^{5,6}$ The behavior of the pressure tensor in the external regions of a configuration was not assigned a priori in the definition of the models; it results from the requirement of self-consistency and from the relevant truncation prescription in phase space.

To carry out a comparison between our differentially rotating models and the observations, we have to specify three dimensionless parameters (the concentration $\Psi$, the rotation strength $\chi$, and the parameter $\bar{b}$ ) and five additional quantities. Three are physical scales (i.e., the radial scale $r_{0}$, the central surface

\footnotetext{
5 Tangentially biased pressure anisotropy in the outer parts of a star cluster is considered to be a natural result of the dynamical evolution of a stellar system within an external tidal field, which induces a preferential loss of stars on radial orbits (this effect has been studied primarily by means of Fokker-Plank and $N$-body simulations; e.g., see Takahashi \& Lee 2000; Baumgardt \& Makino 2003; Hurley \& Shara 2012).

6 Self-consistent models characterized by the presence of tangential anisotropy are rare (see also An \& Evans 2006); so far, the only dynamical model (of $\omega$ Cen) in which the observed tangential anisotropy has been properly taken into account is the descriptive Schwarzschild model constructed by van de Ven et al. (2006).
} 
Table 2

Kinematic Quantities Used to Identify the Dimensionless Parameters of a Model

\begin{tabular}{|c|c|c|c|c|c|c|c|c|}
\hline GC & $\begin{array}{l}\sigma_{0} \\
(1)\end{array}$ & $\begin{array}{c}V_{\max }^{\operatorname{rot}} \\
(2)\end{array}$ & $\begin{array}{c}V_{\max }^{\mathrm{rot}} / \sigma_{0} \\
(3)\end{array}$ & $\begin{array}{l}R_{\mathrm{h}} \\
(4)\end{array}$ & $\begin{array}{c}R_{\max }^{\mathrm{rot}} \\
(5)\end{array}$ & $R_{\text {max }}^{\mathrm{rot}} / R_{\mathrm{h}}$ & $\begin{array}{l}R_{\mathrm{a}} \\
(7)\end{array}$ & $\begin{array}{l}R_{\mathrm{a}} / R_{\mathrm{h}} \\
\quad(8)\end{array}$ \\
\hline$\omega$ Cen & $17.31 \pm 1.72$ & $5.80 \pm 0.32$ & $0.34 \pm 0.04$ & $300.06 \pm 3.51$ & $510.10 \pm 10.21$ & $1.69 \pm 0.04$ & $1035.21 \pm 32.10$ & $3.45 \pm 0.11$ \\
\hline 47 Tuc & $13.06 \pm 1.00$ & $3.26 \pm 0.40$ & $0.25 \pm 0.04$ & $190.22 \pm 3.06$ & $342.40 \pm 5.13$ & $1.80 \pm 0.04$ & $\ldots$ & $\ldots$ \\
\hline M15 & $12.93 \pm 1.06$ & $3.00 \pm 0.63$ & $0.23 \pm 0.05$ & $60.26 \pm 9.70$ & $79.34 \pm 12.54$ & $1.32 \pm 0.30$ & $\ldots$ & $\ldots$ \\
\hline
\end{tabular}

Notes. For each cluster, we report in Column 1: the observed central line-of-sight velocity dispersion $\sigma_{0}$ in km $\mathrm{s}^{-1}$, in Column 2 : the maximum of the line-of-sight rotation profile $V_{\max }^{\text {rot }}$ in $\mathrm{km} \mathrm{s}^{-1}$, in Column 3: the ratio $V_{\max }^{\text {rot }} / \sigma_{0}$, in Column 4: the half-light radius $R_{\mathrm{h}}$ in arcsec from Harris (2010), in Column 5: the position of the maximum of the rotation profile $R_{\max }^{\text {rot }}$ expressed in arcsec, in Column 6: the ratio $R_{\mathrm{max}}^{\text {rot }} / R_{\mathrm{h}}$, in Column 7: the position $R_{\mathrm{a}}$ of the transition from the regime of radial anisotropy to tangential anisotropy in arcsec, and in Column 8: the ratio $R^{\mathrm{a}} / R_{\mathrm{h}}$. A blank space in the last two columns indicates that the desired information is not available from the data. Columns 3, 6, and 8: guide our choice of the three dimensionless parameters that characterize the internal structure of the models.

brightness $\mathrm{SB}_{0}$, and the velocity scale $v_{0}$ ). Finally, the inclination angle $i$ between the rotation axis and the line-of-sight direction, and the distance to the cluster (required to convert the proper motions in $\mathrm{km} \mathrm{s}^{-1}$ ) are also necessary. Such a highly dimensional parameter space is likely to lead to a high degree of degeneracy. Therefore, we decided to separate the modeling procedure into three steps, starting with the focus of interest of this paper-the presence of internal rotation. First, we determine the dimensionless structural parameters by following a few natural selection criteria based on the observed kinematics (see Table 2), then we set the physical scales by means of a few standard statistical fits (this information will be summarized in Tables 3 and 4), and finally we test some properties of the models as predictions in relation to other observational data not used in the first two steps.

Each model in the family is characterized by three dimensionless parameters. Such a three-dimensional parameter space has been explored by paying particular attention to the following general properties of the models: (1) large values of the concentration parameter $\Psi$ determine spatially extended configurations, in terms of the relevant units of length (see VB12 for details); (2) configurations characterized by a given value of concentration and increasing values of the rotation-strength parameter $\chi$ are progressively more compact because of the adopted truncation prescription in phase space; (3) the parameter $\bar{b}$ determines the shape of the line-of-sight rotation profile; in particular, it regulates the radial position of the velocity peak.

\subsection{Dimensionless Parameters}

From Section 2.1.1, it is clear that the GCs under consideration are characterized by significant global internal rotation. Therefore, we start from the observed rotation properties to identify the natural ranges of the three dimensionless parameters. In particular, the parameters should lead to configurations that successfully reproduce the following observations: (1) the observed value of $V_{\max }^{\mathrm{rot}} / \sigma_{0}$, that is, the ratio of the peak of the rotation velocity profile to the central velocity dispersion for the line-of-sight kinematic data; (2) the observed shape of the rotation profile along the line of sight, in particular, the position $R_{\max }^{\text {rot }}$ of the rotation peak (relative to the cluster halflight radius); and (3) the qualitative behavior of the anisotropy profile (when available), in particular, the radial position $R_{\mathrm{a}}$ (relative to the half-light radius) of the transition from radial anisotropy to tangential anisotropy. The relevant observational quantities to be matched by application of the above selection criteria are calculated and listed in Table 2. Specifically, the central velocity dispersion $\sigma_{0}$ and its associated error are average values calculated from the kinematic data within $R_{\mathrm{c}} / 2$; the peak of rotation $V_{\max }^{\mathrm{rot}}$, its radial position $R_{\max }^{\mathrm{rot}}$, and the radial position of the transition from radial anisotropy to tangential anisotropy $R_{\mathrm{a}}$ are calculated by fitting a polynomial to the rotation profile and to the anisotropy profile, in the relevant radial ranges.

Given a set of parameters $(\Psi, \chi, \bar{b})$, the models are projected on the plane of the sky by assuming a known inclination angle $i$, as reported in Table 1 . The projection is performed by sampling from the relevant distribution function a discrete set of $N=$ $2,048,000$ particles and then by performing a rotation of such a discrete system to match the relevant inclination angle. The theoretical kinematic and photometric profiles $^{7}$ are then constructed by following the procedures described in Sections 2.1 and 2.2. The central dispersion $\sigma_{0}$, the maximum of the rotation profile $V_{\max }^{\text {rot }}$, and its position $R_{\max }^{\text {rot }}$ are calculated in view of the above-mentioned selection criteria. As to the morphological aspects, the projected isodensity contours are calculated based on the projected number density distribution. The relevant ellipticity profiles are then constructed by considering the ratio of the principal axes of approximately 100 isodensity contours, corresponding to selected values of the normalized projected number density in the range $\left[0.9,10^{-3}\right]$; smooth profiles are then obtained by performing an average on subsets made of 10-20 individual ellipticity values (depending on the concentration of the configuration).

The dimensionless parameters are varied until the kinematic selection criteria are reasonably met, ${ }^{8}$ that is, until we obtain models consistent within the uncertainties with the observed quantities listed in Table 2.

\subsection{Physical Scales}

Once a set of dimensionless parameters is identified, we proceed to determine the relevant physical scales. This is done by fitting the models to the observed profiles, that is, by minimizing the related $\chi^{2}$. Two fits are performed. With the photometric fit to the surface brightness profile, we determine two scales: the central surface brightness $\mathrm{SB}_{0}$ and the radial scale $r_{0}$ (the scale $r_{0}$ is the standard length scale of King models; e.g., see Equation (A.2) in ZBV12). Once $\mathrm{SB}_{0}$ and $r_{0}$ have been fixed, the velocity scale $v_{0}$ is determined by means of the kinematic fit, which is

\footnotetext{
7 The profiles thus constructed are discrete profiles, which are then interpolated to obtain continuous profiles. The statistical scatter associated with the use of discrete model points is well under control, given the high number of sampling particles considered.

8 Note that the procedure adopted to determine the values of the dimensionless parameters that characterize the internal structure of the models does not allow us to calculate the related formal errors. In any case, we will estimate the range of variation of reasonable models (also in relation to the lack of information on $R_{a}$ for two of the three clusters) by performing a simple exploration of the available parameter space, as described in Section 3.5.
} 
Table 3

Dimensionless Parameters and Physical Scales of the Best-fit Models

\begin{tabular}{|c|c|c|c|c|c|c|c|}
\hline \multirow[t]{2}{*}{$\mathrm{GC}$} & \multicolumn{3}{|c|}{ Dimensionless Parameters } & \multicolumn{3}{|c|}{ Physical Scales } & \multirow{2}{*}{$\begin{array}{c}\text { Dynamical Distance } \\
\qquad d \\
(7)\end{array}$} \\
\hline & $\begin{array}{c}\Psi \\
(1)\end{array}$ & $\begin{array}{c}\chi \\
(2)\end{array}$ & $\begin{array}{c}\bar{b} \\
(3)\end{array}$ & $\begin{array}{c}\mathrm{SB}_{0} \\
(4)\end{array}$ & $\begin{array}{l}r_{0} \\
(5)\end{array}$ & $\begin{array}{l}v_{0} \\
(6)\end{array}$ & \\
\hline$\omega$ Cen & 5.8 & $14.4 \times 10^{-3}$ & 0.040 & $16.43 \pm 0.05$ & $134.54 \pm 1.13$ & $15.87 \pm 0.27$ & $4.11 \pm 0.07$ \\
\hline 47 Tuc & 7.6 & $1.6 \times 10^{-3}$ & 0.008 & $14.30 \pm 0.08$ & $24.41 \pm 0.14$ & $13.35 \pm 0.21$ & $4.15 \pm 0.07$ \\
\hline M15 & 6.8 & $1.6 \times 10^{-3}$ & 0.035 & $14.65 \pm 0.01$ & $13.33 \pm 0.20$ & $12.52 \pm 0.24$ & $10.52 \pm 0.38$ \\
\hline
\end{tabular}

Notes. For each cluster, we list: the concentration parameter $\Psi$ in Column 1, the rotation-strength parameter $\chi$ in Column 2, the $\bar{b}$ parameter in Column 3, the $V$-band central surface brightness $\mathrm{SB}_{0}$ in mag $\operatorname{arcsec}^{-2}$ in Column 4, the radial scale $r_{0}$ in arcsec in Column 5 , the velocity scale $v_{0}$ in $\mathrm{km} \mathrm{s}^{-1}$ in Column 6, and the best-fit dynamical distance $d$ in kpc in Column 7; for the physical scales and the distance, the associated $1 \sigma$ errors are also shown. Note that $r_{0}$ is an intrinsic quantity; it is recorded here in arcseconds, for easier comparison with the observations.

Table 4

Derived Parameters

\begin{tabular}{|c|c|c|c|c|c|c|c|}
\hline $\mathrm{GC}$ & $\begin{array}{c}C \\
(1)\end{array}$ & $\begin{array}{l}R_{\mathrm{c}} \\
(2)\end{array}$ & $\begin{array}{l}R_{\mathrm{h}} \\
(3)\end{array}$ & $\begin{array}{l}r_{\mathrm{tr}} \\
(4)\end{array}$ & $\begin{array}{l}M \\
(5)\end{array}$ & $\begin{array}{c}M / L_{\mathrm{V}} \\
(6)\end{array}$ & $\begin{array}{l}\log \rho_{0} \\
\quad(7)\end{array}$ \\
\hline$\omega$ Cen & $1.27 \pm 0.01$ & $127.8 \pm 1.1$ & $282.5 \pm 2.4$ & $2400.3 \pm 20.2$ & $19.53 \pm 0.16$ & $2.86 \pm 0.14$ & $3.737 \pm 0.034$ \\
\hline 47 Tuc & $1.87 \pm 0.01$ & $24.6 \pm 0.1$ & $162.8 \pm 0.9$ & $1814.9 \pm 10.4$ & $6.23 \pm 0.04$ & $1.69 \pm 0.13$ & $5.090 \pm 0.102$ \\
\hline M15 & $1.94 \pm 0.02$ & $12.9 \pm 0.2$ & $43.7 \pm 0.7$ & $1118.9 \pm 16.8$ & $4.55 \pm 0.07$ & $1.45 \pm 0.05$ & $4.752 \pm 0.130$ \\
\hline
\end{tabular}

Notes. For each cluster we provide the structural parameters derived for the best-fit models: $(1)$ the concentration parameter $C=\log \left(r_{\mathrm{tr}} / R_{\mathrm{c}}\right)$, (2) the projected core radius $R_{\mathrm{c}}$ in arcsec, (3) the projected half-mass radius $R_{\mathrm{h}}$ in arcsec, (4) the truncation radius $r_{\mathrm{tr}}$ in arcsec, (5) the total mass of the cluster $M$ in units of $10^{5} M_{\odot},(6)$ the $V$-band mass-to-light ratio in solar units, and (7) the logarithm of the central mass density $\rho_{0}$ in units of $M_{\odot} \mathrm{pc}^{-3}$.

performed by minimizing a combined $\chi^{2}$ defined as the sum of the contributions from the line-of-sight rotation and the lineof-sight velocity dispersion profiles. Finally, the mass-to-light ratio is directly connected to the central surface brightness by the following relation: $M / L_{\mathrm{V}}=\hat{\Sigma}(0) 10^{\mathrm{SB}_{0} / 2.5}$, where $\hat{\Sigma}(0)$ denotes the central surface density expressed in the relevant units. The details of the fitting procedure and of the calculation of the errors are given in Appendix B of ZBV12.

\subsection{Dynamical Distance Measurement}

The kinematic information associated with the proper motions is used to measure the distance to the cluster. The relation between proper motions $\mu$ measured in mas $\mathrm{yr}^{-1}$ and proper motions $v$ expressed in $\mathrm{km} \mathrm{s}^{-1}$ is

$$
\left[\frac{v}{\mathrm{~km} \mathrm{~s}^{-1}}\right]=4.74\left[\frac{d}{\mathrm{kpc}}\right]\left[\frac{\mu}{\mathrm{mas} \mathrm{yr}^{-1}}\right],
$$

where $d$ is the distance from the observer to the GC. Therefore, with all the dimensionless parameters and physical scales fixed from the previous analysis, we obtain a best-fit distance $d$ (hereafter referred to as dynamical distance) by a combined fit to the observed tangential $\sigma_{t}$ and radial $\sigma_{R}$ velocity dispersion profiles (i.e., by minimizing a combined $\chi^{2}$ defined as the sum of the contributions of the two velocity dispersion profiles in the plane of the sky).

\subsection{Predicted Profiles}

At this stage for a given cluster, the model and the relevant scales have all been determined. A number of other observable quantities are then predicted and can be compared to the available observations. In particular, we wish to include in this category the following quantities: the anisotropy profile $\sigma_{t} / \sigma_{R}$, the proper-motion mean-velocity profiles $V_{t}$ and $V_{R}$, the ellipticity profile $\varepsilon$, and the two-dimensional structure of the isodensity contours, which need not be perfect ellipses.

\subsection{Exploration of Parameter Space}

The procedure adopted for the selection of a rotating model gives priority to the kinematic data, which are usually affected by large uncertainties and often do not cover a sufficiently wide radial extent. Therefore, it is important to check whether the selection procedure might be improperly sensitive to these uncertainties. In order to do so, we perform an exploration of the available dimensionless parameter space (Section 3.1) by estimating what range of parameters would be consistent with the uncertainties associated with the kinematic observed quantities listed in Table 2.

For each selected model that meets the kinematic criteria, we calculated the physical scales by means of the fits described in Sections 3.2 and 3.3. The best-fit model is taken to be the one that minimizes the total $\chi^{2}$ (defined as the sum of the calculated $\chi^{2}$ for the photometric, kinematic, and distance fits). As an example of this procedure, in Figure 2 we show three different models for $\omega$ Cen, characterized by different values of the $V_{\max }^{\text {rot }} / \sigma_{0}$ parameter: $0.28,0.34$, and 0.36 , respectively. The three models give comparable results for the kinematic profiles, very similar results for the photometric profiles, and, most importantly, they all give similar trends in the predicted ellipticity profiles, as shown in Figure 5. Therefore, we conclude that our selection procedure can be considered to be sufficiently stable and reliable.

Moreover, we recall that the kinematic data on the plane of the sky for 47 Tuc and M15 (see Sections 5 and 6, respectively) are not radially extended enough to allow us to determine the complete shape of the anisotropy profile. Therefore, in these cases, the $R_{\mathrm{a}}$ scale, which marks the radial position of the transition from radial to tangential anisotropy, cannot be used as an additional criterion for the selection of the dimensionless parameters. However, the exploration of the parameter space just described already includes models with varying $R_{\mathrm{a}}$, because the shape of the anisotropy profile is 

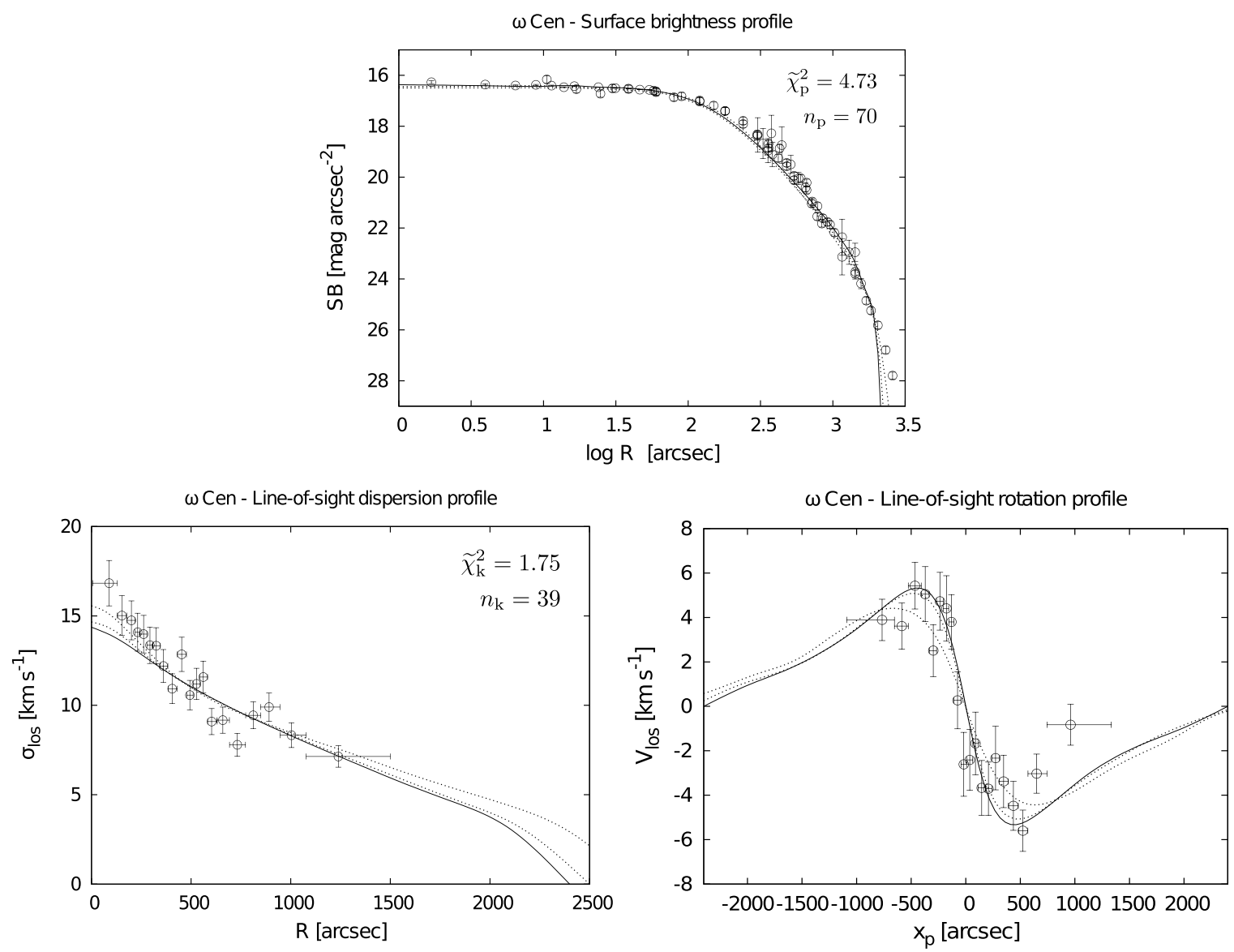



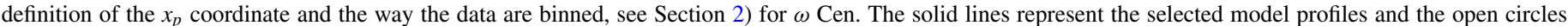

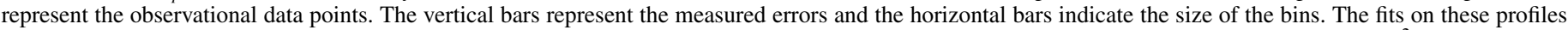



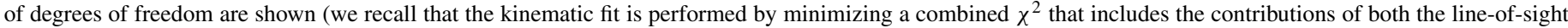

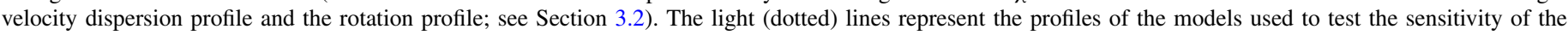
selection procedure to the specific choice of kinematical parameters on which the procedure is based, as described in Section 3.5 .

directly intertwined with the observational parameters taken into consideration.

\section{4. $\omega$ Cen}

The GC $\omega$ Cen is the cluster for which the most complete photometric and kinematic data are available. In particular, the data set considered in this paper consists of 1868 lineof-sight velocities, 2740 ground-based proper motions, and 72,970 HST proper motions (see Appendix A). The kinematic profiles that we have constructed from these data extend out to $\approx 0.5 r_{\text {tr }}$; therefore, a thorough comparison between models and observations can be carried out.

\subsection{Photometry and Kinematics}

In general, the selected model is in satisfactory agreement with the surface brightness profile and the line-of-sight kinematic profiles, as shown in Figure 2. For the photometric profile, the model reproduces well the central regions and the intermediate parts, but it underestimates the last two data points. For the line-of-sight kinematic profiles, the model is able to reproduce simultaneously the shape of the rotation profile and the shape of the velocity dispersion profile, with one important failure: the central values (inside $\approx 200$ arcsec) of the line-of-sight velocity dispersion are severely underestimated by our model.
It is interesting to note that any quasi-Maxwellian dynamical model applied to $\omega$ Cen is unable to reproduce the cuspy behavior observed in the central regions (e.g., see the application of spherical King models and of spherical Wilson models presented by MLvdM05 in their Figure 11; see also the fit by means of the rotating Wilson 1975 model performed by Sollima et al. 2009). In this respect, radially biased anisotropic models appear to perform better (in particular, see the application of the $f^{(v)}$ models discussed by ZBV12). On the one hand, this feature has sometimes been considered as evidence for the presence of a central IMBH (see Noyola et al. 2008). On the other hand, the same feature may indicate that $\omega$ Cen, because of its relatively long relaxation times (see Table 1), is only partially relaxed and characterized by a higher degree of radial anisotropy with respect to the case of more relaxed stellar systems, as suggested by Figure 3 (see also van der Marel \& Anderson 2010). A more detailed discussion of this issue is postponed to Section 7, where a comparison among models with different anisotropy profiles is presented. Curiously, even though (see Appendix B) the lineof-sight data indicate high rotation in the very central regions ( $R<0.5 R_{\mathrm{c}}$ ), which is naturally interpreted as the signature of a complex rotating central structure, this does not appear to affect the quality of our results on the rotation profile; in fact, the selected model reproduces the central part of the line-of-sight rotation curve surprisingly well (see Figure 2). 

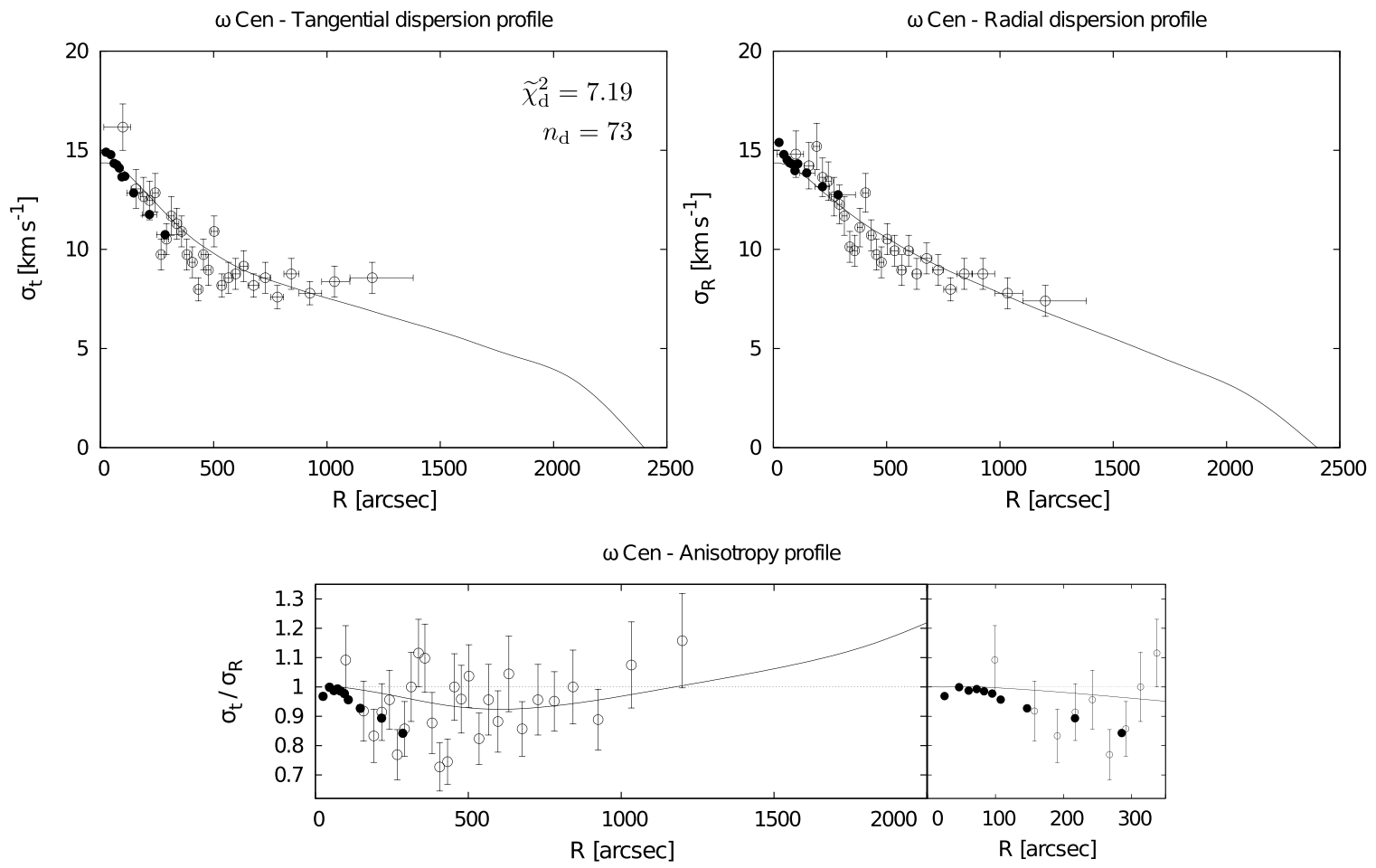

Figure 3. Top panels illustrate the fit to the proper-motion dispersion profiles along the projected tangential and radial directions for $\omega$ Cen; this fit determines the dynamical distance $d$. The associated reduced $\chi^{2}$ and the number of degrees of freedom are shown. The bottom panels show the predicted anisotropy profile against the available data, on the large (left) and small (right) radial scale. Solid lines represent the model profiles, open circles represent the observational data points from van Leeuwen et al. (2000), and black dots represent the data from Anderson \& van der Marel (2010). Vertical bars indicate the measured errors and horizontal bars indicate the size of the bins.

In addition, the model identified by our procedure is able to reproduce all three components of the projected velocity dispersion tensor (both along the line of sight and on the plane of the sky; see Figure 3). Interestingly, the shape of the observed anisotropy profile built from the proper-motion dispersions is consistent with the general properties of the selected model, which is characterized by isotropy in the central region, weak radial anisotropy in the intermediate region, and tangential anisotropy in the outer parts. The transition between the region characterized by radial anisotropy and the region characterized by tangential anisotropy takes place at $R \approx 1200 \mathrm{arcsec}$. The data indeed show signs of radial anisotropy in the intermediate region (note that our model predicts a degree of radial anisotropy lower than the one observed) and tangential anisotropy outside $R \approx 1000 \mathrm{arcsec}$. The existence of tangential anisotropy in the present study is consistent with the results of previous investigations, namely, van de Ven et al. (2006) and van der Marel \& Anderson (2010; see their Figure 6). We wish to emphasize that such behavior of the anisotropy profile in the outer parts is a natural property of the family of models that form the basis of the present work.

Finally, we can also compare the rotation on the plane of the sky predicted by the model with the observed mean-velocity profiles along the tangential and radial directions. Figure 4 shows that the tangential proper-motion mean-velocity profile $V_{t}(R)$ is well reproduced by the model, confirming the presence of differential rotation. In the radial direction, the model predicts a flat profile with vanishing velocity; in the external regions $(R>1000 \operatorname{arcsec})$, the observed proper-motion mean velocity in the radial direction reaches a value of $V_{\mathrm{R}} \approx 5 \mathrm{~km} \mathrm{~s}^{-1}$, indicating the presence of a systematic expansion ascribed to systematic errors in the measurement procedures (van Leeuwen et al. 2000). At this point, we should also recall that the procedure used to measure the proper motions removes any sign of solid-body rotation in the plane of the sky; therefore, we apply to the data a correction to recover the solid-body mean-velocity component, following van de Ven et al. (2006), as discussed in Appendix A. This fact introduces some uncertainties in the final profiles and might account for some of the discrepancies between the model and the observed proper-motion meanvelocity profiles.

In conclusion, aside from the inner cusp problem, the generally good agreement between model and proper-motion meanvelocity and velocity dispersion profiles is quite remarkable, because the model was selected only to match the velocity-todispersion ratio along the line of sight $V_{\max }^{\text {rot }} / \sigma_{0}$, the location of the peak in the rotation profile along the line of sight $R_{\max }^{\text {rot }}$, and the location of the transition from radial to tangential anisotropy in the plane of the sky.

\subsection{Dynamical Distance}

Rescaling the model profiles to match the observed propermotion dispersion profiles allows us to derive an estimate for the distance of the cluster (see Section 3.3). The dynamical distance obtained for $\omega$ Cen is $d=4.11 \pm 0.07 \mathrm{kpc}$, with an associated reduced $\widetilde{\chi}_{\mathrm{d}}^{2}=7.19$. This distance is significantly smaller than the distance estimated with photometric methods (e.g., $d=5.2 \pm 0.7 \mathrm{kpc}$ from Harris 2010) and also smaller than other estimates obtained by means of the application of different dynamical models (e.g., $d=4.70 \pm 0.06 \mathrm{kpc}$ from van der Marel \& Anderson 2010; $d=4.8 \pm 0.3 \mathrm{kpc}$ from van de Ven et al. 2006).

As also noted by van de Ven et al. (2006), a low value of the distance is expected when either the line-of-sight velocity dispersion is underestimated or the proper-motion dispersion is 

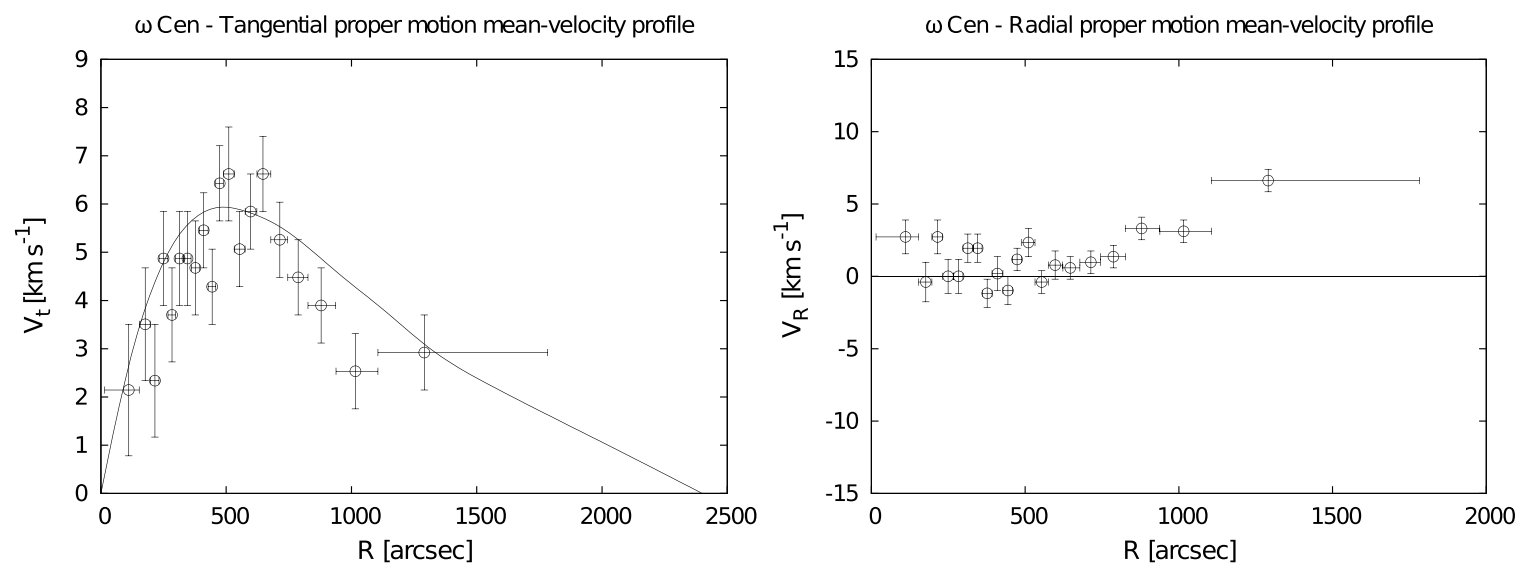

Figure 4. Predicted proper-motion mean-velocity profiles along the tangential and radial directions for $\omega$ Cen. The solid lines represent the model profiles and the open circles represent the observational data points. The vertical bars indicate the measured errors and the horizontal bars indicate the size of the bins. Note that the data give a hint of a possible overall expansion, which is obviously not present in the model.

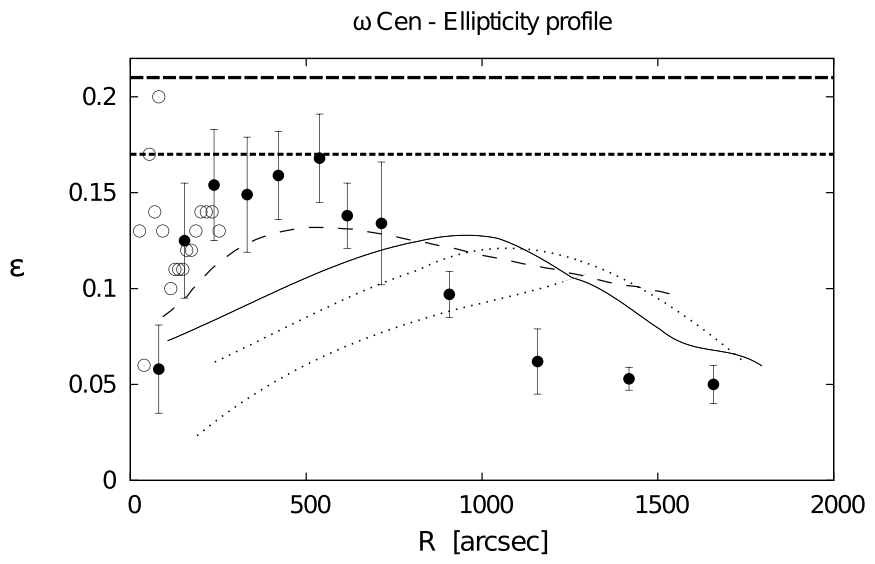

Figure 5. Ellipticity profile for $\omega$ Cen. The open circles mark the observed ellipticities from Anderson \& van der Marel (2010) and the black dots mark those from Geyer et al. (1983). The solid line represents the predicted profile derived from the rotating axisymmetric model proposed in this paper, whereas the thin dotted curves correspond to the models used to test the sensitivity of the selection procedure (see Section 3.5). The dotted and dashed horizontal lines indicate the average values from WS87 and CC10, respectively. Finally, the long-dashed line represents the ellipticity profile for the best-fit rotating Wilson (1975) model, from Sollima et al. (2009); see discussion in Section 7.1.

overestimated. In our case, it is clear from the previous section and from Figure 2 that our dynamical model underestimates the central value of the line-of-sight velocity dispersion. Therefore, our distance estimate is affected by a systematic bias (reflected also by the high value of the reduced $\chi^{2}$ ). The dynamical distances obtained by van der Marel \& Anderson (2010) and van de Ven et al. (2006) are based on a Jeans model and on an orbit-based model, respectively; previous studies based on the application of quasi-Maxwellian dynamical models, such as spherical King or spherical Wilson models, do not report distance estimates for this object.

\subsection{Deviations from Spherical Symmetry}

The selected axisymmetric model is associated with a welldefined ellipticity profile, which is the morphological counterpart to the presence of rotation. The comparison with the corresponding observed profile is illustrated in Figure 5; the open circles represent the profile from Anderson \& van der Marel (2010), the black dots represent the profile from Geyer et al. (1983), and the solid line represents the profile derived from our

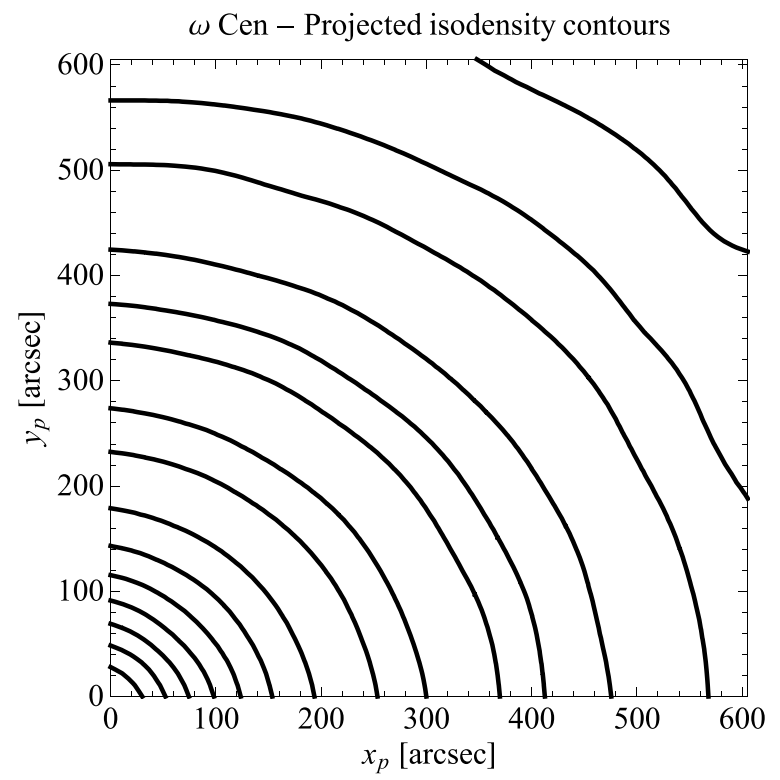

Figure 6. Predicted projected isodensity contours for $\omega$ Cen. The contours are calculated in the first quadrant of the plane of the sky and correspond to selected values of the projected number density (normalized to the central value) in the range $\left[0.9,10^{-2}\right]$. The area represented in the figure covers a square of side length approximately equal to $2 R_{h}$.

model. The two observed profiles are consistent in the sampled radial range, except for the innermost region $(R<100$ arcsec $)$ where a large scatter dominates the data of Anderson \& van der Marel (2010). For completeness, in Figure 6, we present the projected isodensity contours predicted by our model, which show the two-dimensional deviations from sphericity. The contour shapes are of interest for future comparisons with observations based on more detailed morphological studies and may provide an important clue to distinguish between different dynamical models (see, e.g., VB12).

The model ellipticity profile is characterized by a general trend similar to that of the Geyer et al. (1983) measurements, but it predicts the peak of maximum flattening too far out, at about $R \approx 1000$ arcsec. If we calculate the average ellipticity in the radial range covered by the data, then we find an average flattening associated with the selected model $(\varepsilon=0.10)$ in agreement with the observed one $(\varepsilon=0.12 \pm 0.02)$. In other words, we are led to conclude that the observed deviations from 

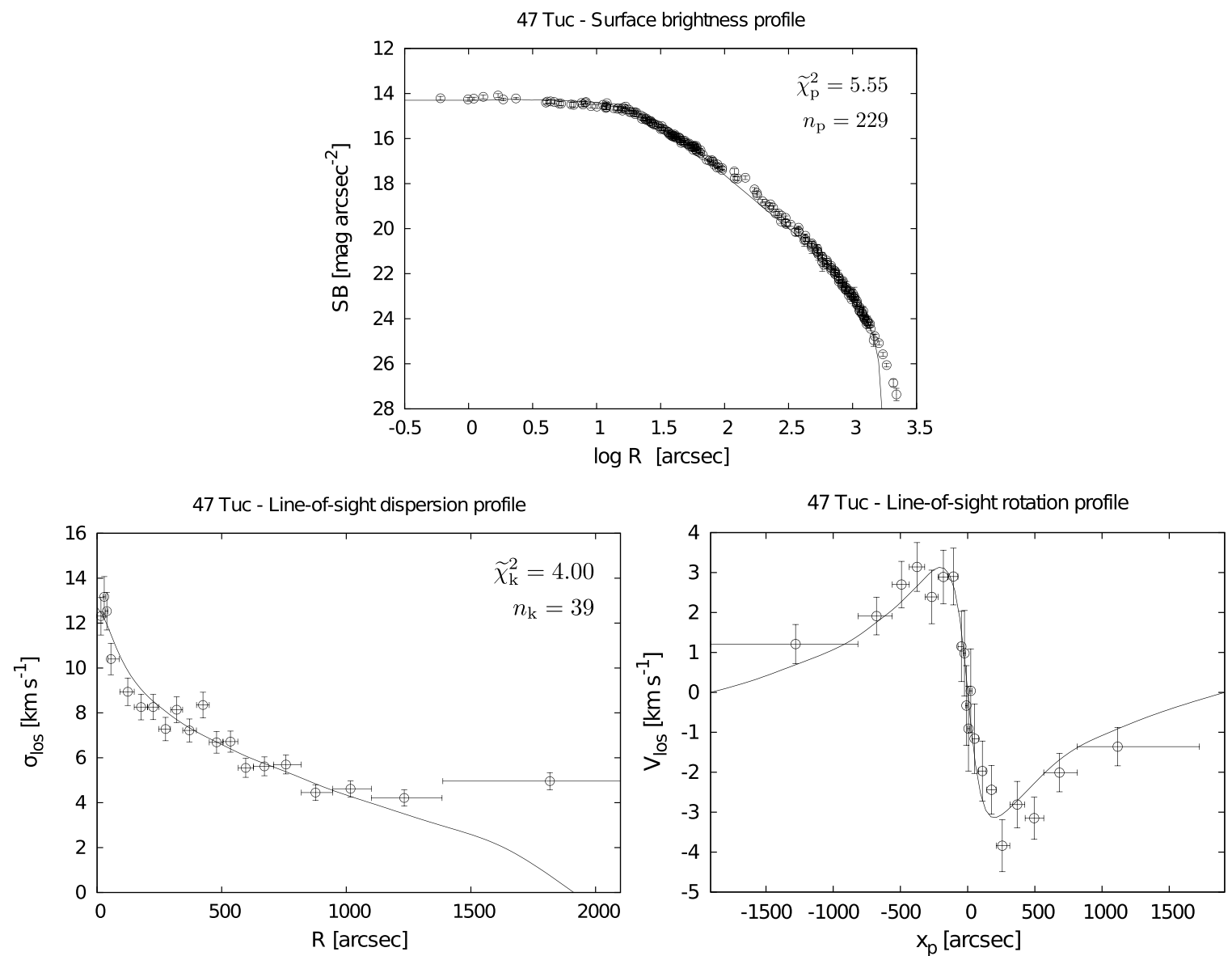

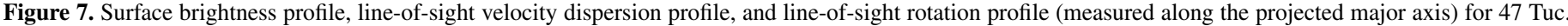
The associated photometric and kinematic reduced $\chi^{2}$ and the number of degrees of freedom are shown. For a description of the symbols and curves, see Figure 2.

sphericity are likely to originate in the presence of internal rotation. In Section 7, we will argue that the discrepancy between the predicted and observed ellipticity profiles is likely to be related to the complex nature of $\omega$ Cen, in particular its conditions of partial relaxation and the interplay between rotation and anisotropy in velocity space. In this respect, we expect that our models of quasi-relaxed stellar systems will perform better for GCs characterized by shorter relaxation times (such as 47 Tuc and M15).

\section{47 Tuc}

For the GC 47 Tuc, the data set consists of 2476 line-of-sight velocities and 12,974 HST proper motions (see Appendix A). The line-of-sight kinematical data cover the full radial extent of the cluster, out to approximately the truncation radius. In turn, the proper-motion data are limited to a disk of radius $4 R_{\mathrm{c}}$.

\subsection{Photometry and Kinematics}

As illustrated in Figure 7, the surface brightness profile and the line-of-sight rotation and velocity dispersion profiles are well reproduced by the selected model. In particular, the rotation profile is well matched throughout the extent of the cluster, showing clearly the position of the maximum rotation velocity, the characteristic rigid rotation behavior in the central region, and the relatively sharp decrease in the outer parts. The observed line-of-sight velocity dispersion profile is characterized by one data point at $R \gtrsim 1800$ arcsec deviating from the model profile. A corresponding discrepancy is found also for the surface brightness profile, at approximately the same radial position (the last four photometric data points). These two features may be interpreted in terms of the population of "potential escapers" resulting from the tidal interaction between the cluster and the host Galaxy (see Küpper et al. 2010; Lane et al. 2012).

As to the proper-motion data, the relevant profiles, although limited to the central region, show a satisfactory agreement with the model predictions (see Figure 8). In the intermediate regions $(50 \lesssim R \lesssim 1000$ arcsec), the model predicts weak radial anisotropy and tangential anisotropy in the outer parts. It would be interesting to acquire more spatially extended proper-motion measurements to confirm this prediction (in line with the results obtained for the anisotropy profile of $\omega$ Cen; see Figure 3).

Rotation in the plane of the sky is not available from the proper-motion data set of McLaughlin et al. (2006). However, proper-motion rotation has been measured by Anderson \& King (2003), using HST and considering background stars of the Small Magellanic Cloud as an absolute reference frame. The observed rotation corresponds to a velocity of $4.97 \pm 1.17 \mathrm{~km} \mathrm{~s}^{-1}$ (based on the assumed distance of $4.5 \mathrm{kpc}$ ) at a projected radius of 5.7 arcmin (corresponding approximately to the position of the rotation peak). Within the uncertainties, this is consistent with our model, which predicts a value of $4.13 \mathrm{~km} \mathrm{~s}^{-1}$ at 5.7 arcmin.

\subsection{Dynamical Distance}

The comparison of the observed proper-motion dispersion profiles with the model predictions allows us to derive an 

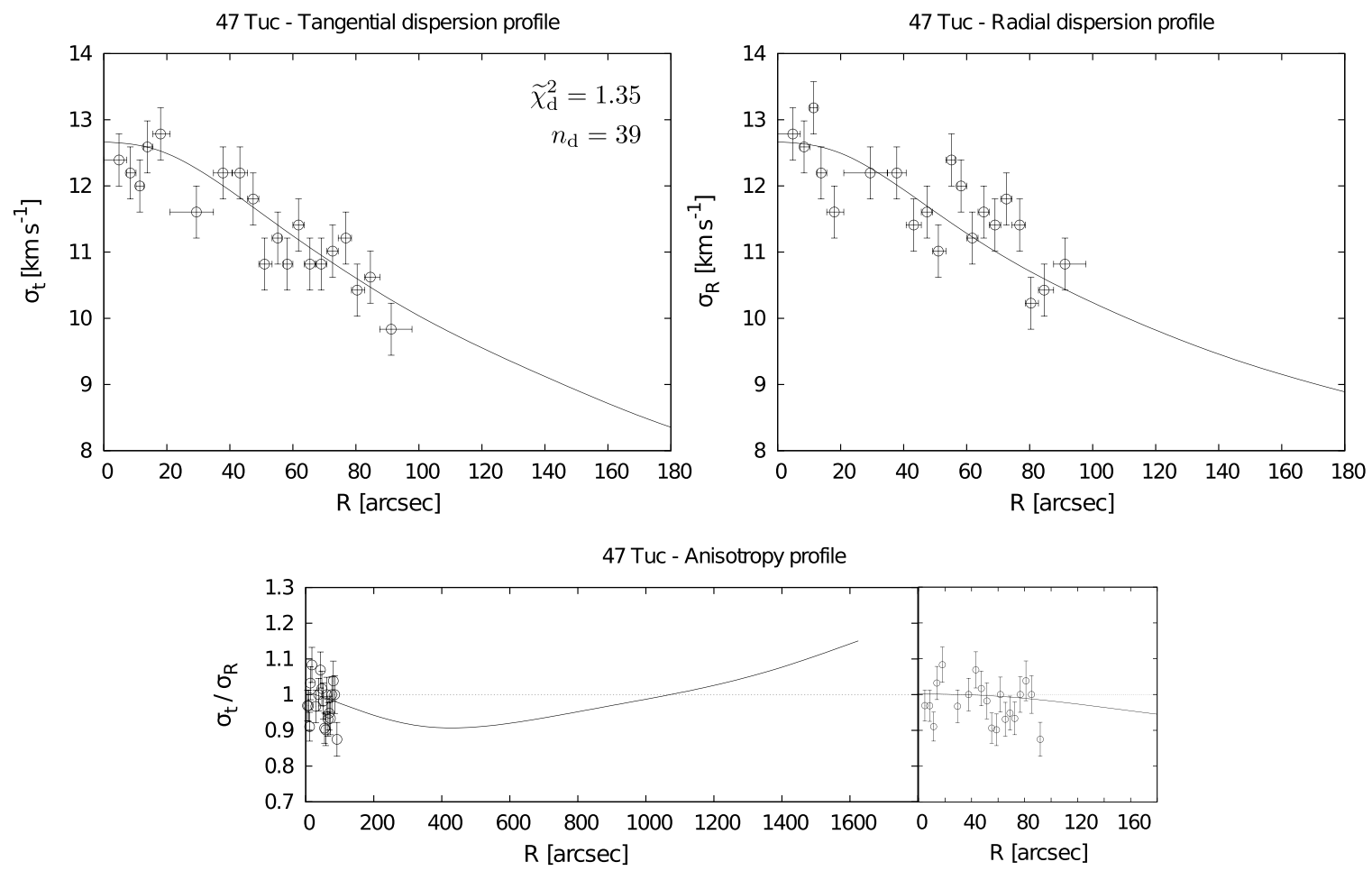

Figure 8. Tangential dispersion, radial dispersion, and anisotropy profile for 47 Tuc. The associated reduced $\chi^{2}$ and the number of degrees of freedom are shown. For a description of the symbols and curves, see Figure 3.

estimate of the distance to the cluster (see Section 3.3). For 47 Tuc, the best-fit distance is $d=4.15 \pm 0.07 \mathrm{kpc}$, with associated reduced $\tilde{\chi}_{\mathrm{d}}^{2}=1.35$ inside the corresponding $90 \%$ confidence interval (CI). This value is consistent with the dynamical distance reported by McLaughlin et al. (2006) of $d=4.02 \pm 0.35 \mathrm{kpc}$, measured from the same proper-motion data set used in the present work, under the simple assumptions of spherical symmetry, isotropy, and absence of internal rotation. Our value is lower than the standard value of $d=4.5 \pm 0.2 \mathrm{kpc}$ reported in the Harris catalog (Harris 2010) and lower than other distance estimates obtained by means of photometric methods, such as main-sequence fitting, RR Lyrae, and whitedwarf cooling sequence fitting (for a recent summary of results, see Table 1 of Woodley et al. 2012 or Bono et al. 2008).

\subsection{Deviations from Spherical Symmetry}

Figure 9 shows the ellipticity profile predicted by our model plotted together with the ellipticity data available for 47 Tuc. In this cluster, the deviations from spherical symmetry are naturally explained by the selected model with a surprising degree of accuracy. In fact, the ellipticity profile derived by our model reproduces the radial variation of the observed ellipticity over the entire spatial range covered by the data (the flattening of 47 Tuc increases from a value of $\varepsilon \approx 0$ to a maximum value of $\varepsilon \approx 0.12$ at $R \approx 450$ arcsec). We recall that the ellipticity profile associated with the selected self-consistent model is a structural property completely determined by the dimensionless parameters and physical scales identified during the model selection procedure. In this case, we can thus state with confidence that internal rotation is the physical ingredient responsible for the observed global deviations from spherical symmetry. In this respect, we emphasize that the relation between the shapes of the rotation profile and the ellipticity profile is highly nontrivial; in particular, the peak of the rotation

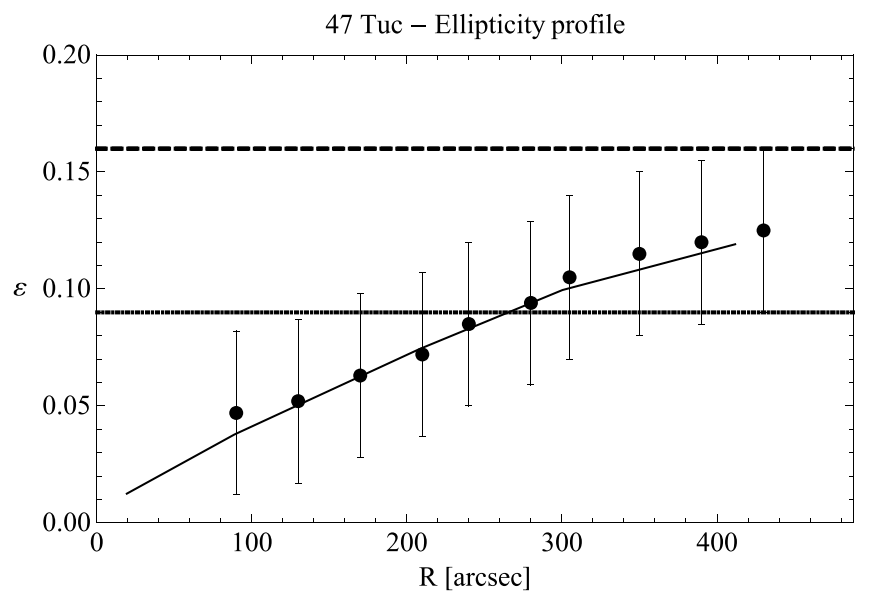

Figure 9. Ellipticity profile for 47 Tuc. The black dots mark the observed ellipticities presented by WS87 and the solid line represents the profile derived from our axisymmetric rotating model. The dotted and dashed horizontal lines indicate the average values reported by WS 87 and CC10, respectively.

profile does not correspond to a peak in the ellipticity profile (at odds with what is often believed; e.g., Meylan \& Mayor 1986).

\section{M15}

The studies of the GC M15 are largely focused on its central region. In fact, the cluster is believed to be in a post-corecollapse phase and mass segregation is thought to play a role in its dynamics. In particular, the sharp gradient of the central luminosity is thought to be the result of the dynamical evolution of the cluster (see, e.g., Baumgardt \& Makino 2003; Murphy et al. 2011) or of the presence of a central IMBH (see, e.g., Gerssen et al. 2002). The available kinematic data are limited to 

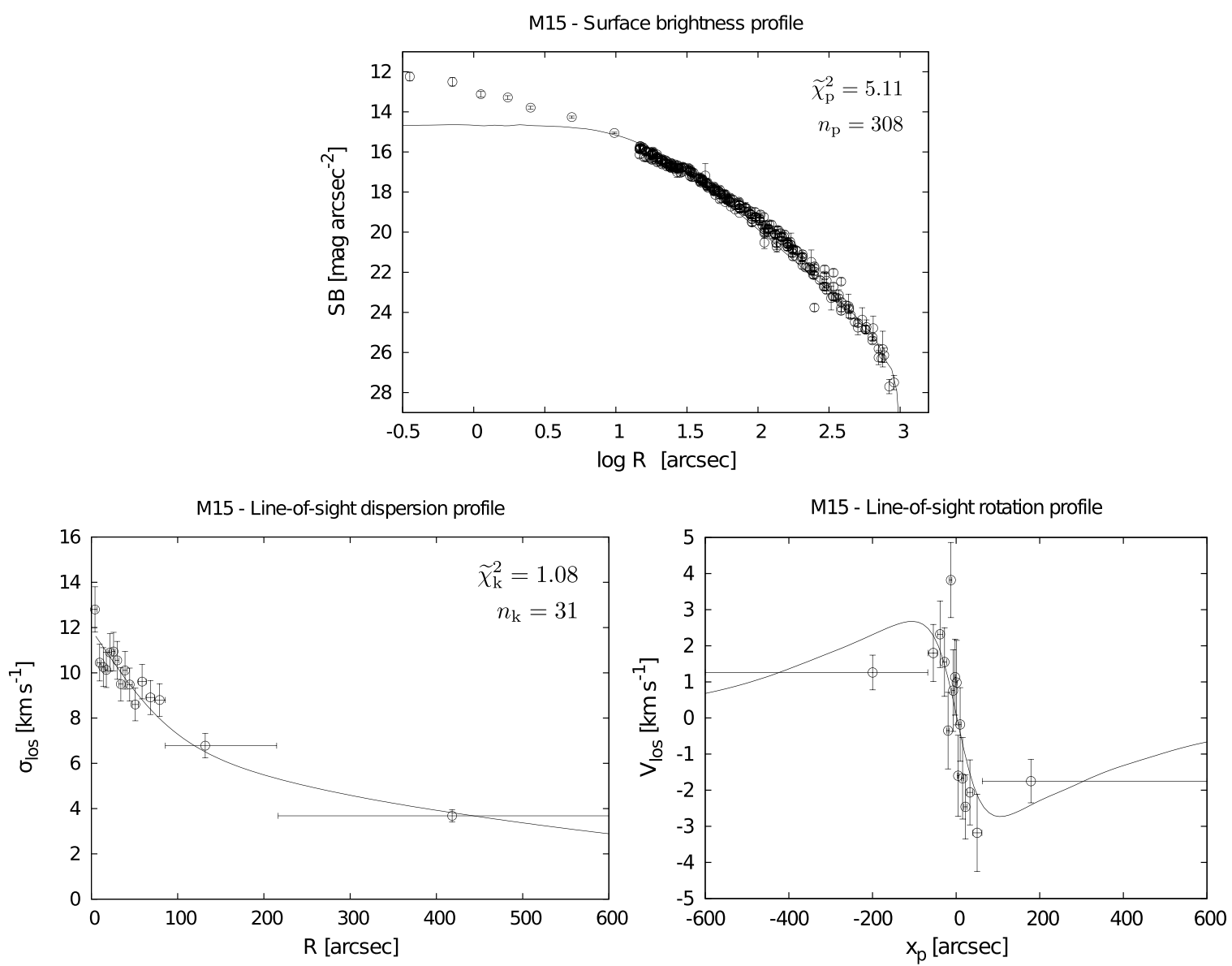

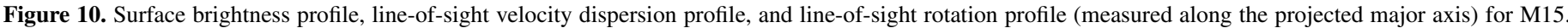
The associated photometric and kinematic reduced $\chi^{2}$ and the number of degrees of freedom are shown. For a description of the symbols and curves, see Figure 2.

the central regions and consist of 1777 line-of-sight velocities and 703 HST proper motions (see Appendix A).

\subsection{Photometry and Kinematics}

Remarkably, except for the most central region, the selected model offers a good description of both the line-of-sight kinematic profiles and the surface brightness profile (see Figure 10). The line-of-sight velocity dispersion profile is reproduced by the model out to the last available bin, located at approximately $0.5 r_{\text {tr }}$.

As to the line-of-sight rotation profile, a large scatter is present in the central regions due to the large measurement errors, which have an average value of $3.79 \mathrm{~km} \mathrm{~s}^{-1}$ (significantly higher than the average errors of $\omega$ Cen and 47 Tuc: $1.98 \mathrm{~km} \mathrm{~s}^{-1}$ and $2.29 \mathrm{~km} \mathrm{~s}^{-1}$, respectively). Unfortunately, the kinematic data set does not sample the region where the peak of the rotation curve is expected. More accurate and better distributed line-of-sight velocity measurements would be required to build a more reliable and complete rotation profile. However, it is interesting to note that the rotation profile in the central regions, characterized by a solid-body behavior, is well accounted for by the model, although high rotation is detected in the center and interpreted as a signature of the presence of a decoupled rotating core (see Appendix B).

For the proper motions, given the small number of data and the low accuracy of the measurements, we decided to divide the sample into only four bins to avoid excessive statistical noise; the relevant profiles are illustrated in Figure 11. Such profiles can be used to constrain the kinematic behavior of the cluster only in relation to the very central regions. In turn, the selected model leads to specific predictions of the anisotropy profile in the intermediate and outer parts of the object, which are expected to first show weak radial anisotropy and then tangential anisotropy. Unfortunately, for this object no information about the rotation on the plane of the sky is yet available.

\subsection{Dynamical Distance}

The dynamical distance obtained from the procedure described in Section 3.3 yields a distance of $d=10.52 \pm$ $0.38 \mathrm{kpc}$, with a reduced $\widetilde{\chi}_{\mathrm{d}}^{2}=0.41$, inside the corresponding $90 \%$ CI. This is consistent with the kinematic distance obtained by McNamara et al. (2004) of $d=9.98 \pm 0.47 \mathrm{kpc}$ and the value obtained by van den Bosch et al. (2006) of $d=10.3 \pm 0.4 \mathrm{kpc}$; these two estimates are based on the same proper-motion data set as considered in the present work. In particular, the value obtained by McNamara et al. (2004), which is based on the simplifying assumptions of spherical symmetry, isotropy in velocity space, and no rotation, is lower than the value obtained in the present paper and the one obtained by van den Bosch et al. (2006, in which anisotropy, rotation, and flattening are taken into account). Moreover, our distance is in agreement with other distance estimates based on photometric methods, such as the one reported in the Harris catalog (Harris 2010) of $d=10.4 \pm 0.8 \mathrm{kpc}$. In this case, the conclusion drawn by Bono et al. (2008) — that distances obtained from kinematic 

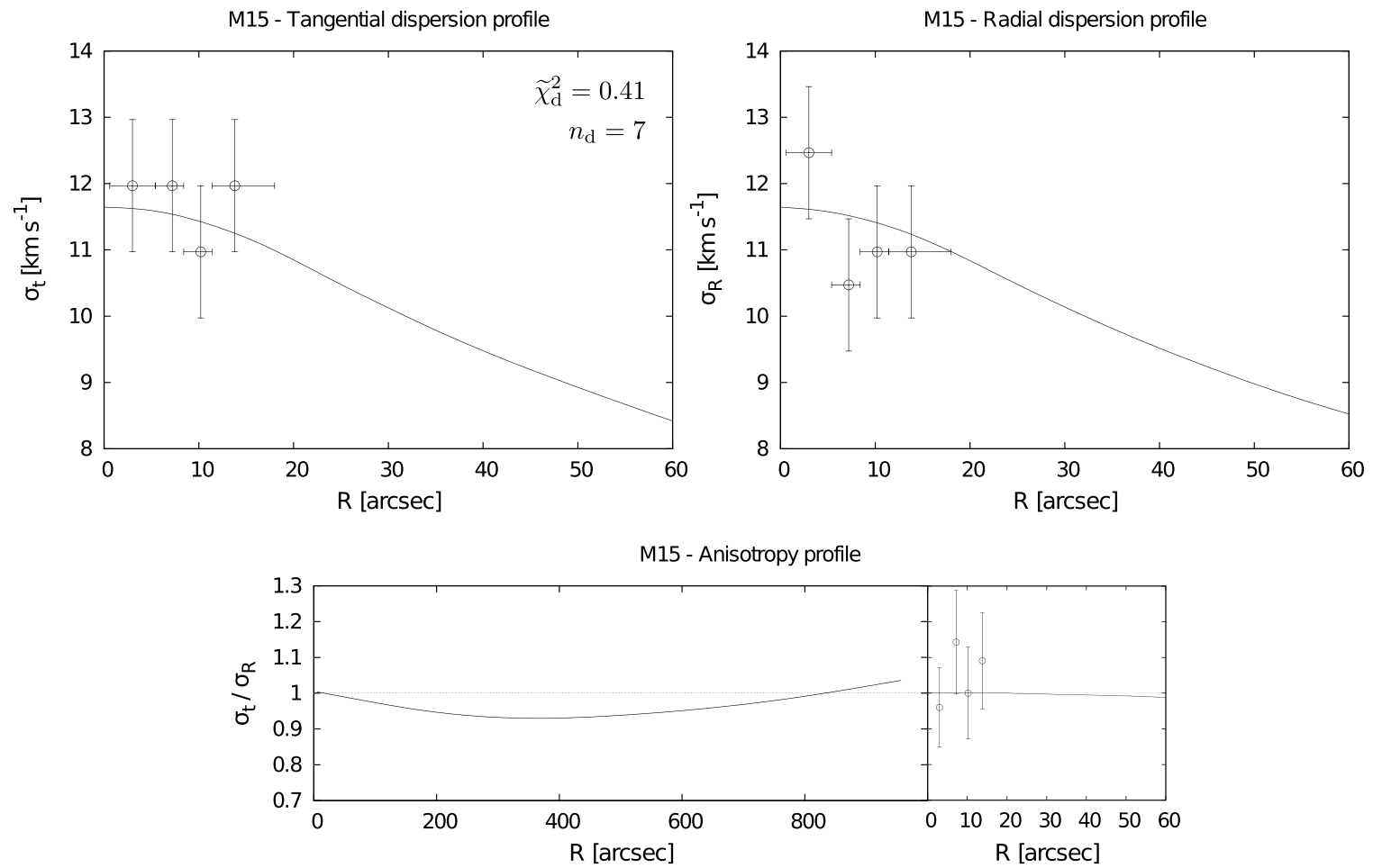

Figure 11. Tangential dispersion, radial dispersion, and anisotropy profile for M15. The associated reduced $\chi^{2}$ and the number of degrees of freedom are shown. For a description of the symbols and curves, see Figure 3.

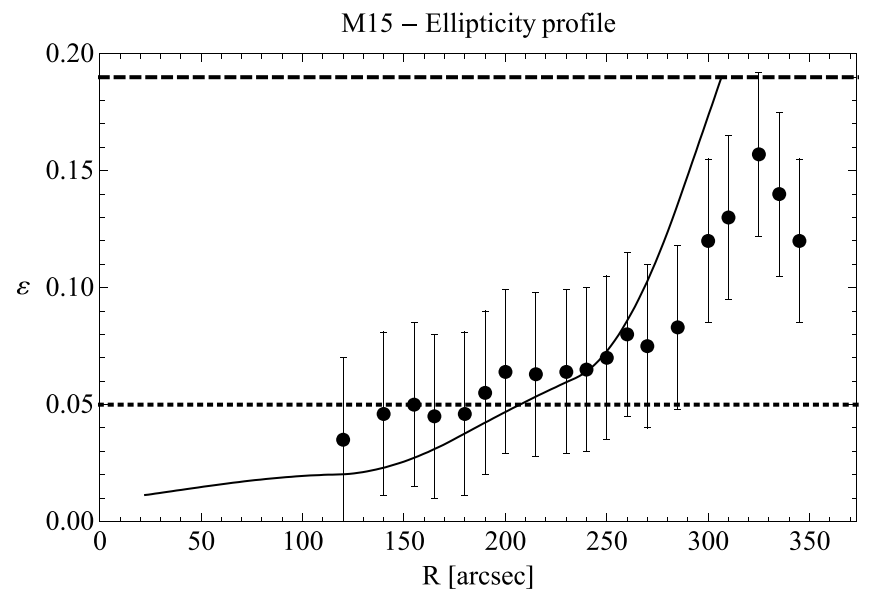

Figure 12. Ellipticity profile for M15. For a description of the symbols and curves, see Figure 9.

data are systematically lower than distances obtained from other methods-does not hold.

\subsection{Deviations from Spherical Symmetry}

The comparison between the observed and the predicted ellipticity profiles is illustrated in Figure 12. Our model predicts an ellipticity close to zero in the very central regions and an increase of the flattening thereafter, consistent with the observations. In particular, we note that the model profile seems to overlap smoothly with the observed profile in the region sampled by the data. Moreover, the observed average flattening is consistent with the value predicted by our model. We thus conclude that our dynamical model, and consequently the presence of internal rotation, can naturally explain the observed deviations from sphericity of M15.

\section{DISCUSSION}

\subsection{Partially Relaxed versus Well-relaxed Clusters}

The three GCs under consideration are known to be in different evolutionary states. In fact, the core relaxation time of $\omega$ Cen is significantly higher than the relaxation times of 47 Tuc and M15 (see Table 1). This suggests that $\omega$ Cen should be in a partially relaxed state, whereas 47 Tuc and M15 can be considered to be well-relaxed clusters.

In the case of $\omega$ Cen, we argue indeed that the main discrepancies noted between our model and observations are associated with the condition of partial relaxation of the cluster. Our model is unable to describe the cuspy behavior of the velocity dispersion profile in the central regions (inside $\approx$ 300 arcsec). In Figure 13, we compare our quasi-relaxed model with the best fit (spherical, nonrotating, and nontruncated) $f^{(v)}$ model from ZBV12 and the best-fit axisymmetric, rotating Wilson (1975) model from Sollima et al. (2009). The top panel shows that in the central region $\left(R \lesssim 500 \operatorname{arcsec} \approx 2 R_{\mathrm{h}}\right)$, the gradient of the line-of-sight dispersion profile depends strongly on the assumed model: the steepest gradient is associated with the spherical $f^{(v)}$ model, which is the model characterized by the strongest radial anisotropy. Note that the spherical $f^{(v)}$ model and the rotating Wilson (1975) model both miss the feature of tangential anisotropy in the outer regions altogether. This is further illustrated by the bottom panel, which shows the intrinsic anisotropy parameter $\beta=1-\left(\sigma_{\theta}^{2}+\sigma_{\phi}^{2}\right) / 2 \sigma_{r}^{2}$ profile evaluated along the equatorial plane. Indeed, the rotating models constructed in VB12 and applied in this paper are characterized by isotropy in the central regions and only weak radial anisotropy in the intermediate radial range because they assume that the stellar system is quasi-relaxed.

In Figure 5, the ellipticity profile predicted by our model is compared with the profile presented by Sollima et al. (2009) 
$\omega$ Cen - Line-of-sight dispersion profile

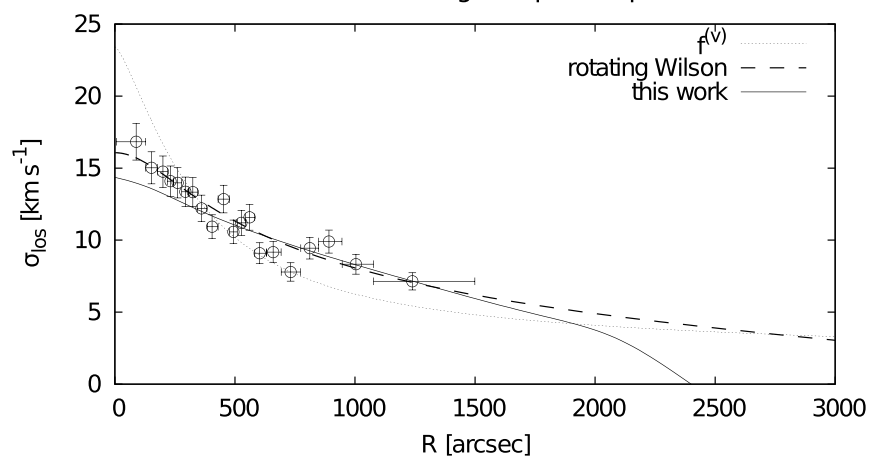

$\omega$ Cen - intrinsic anisotropy profile

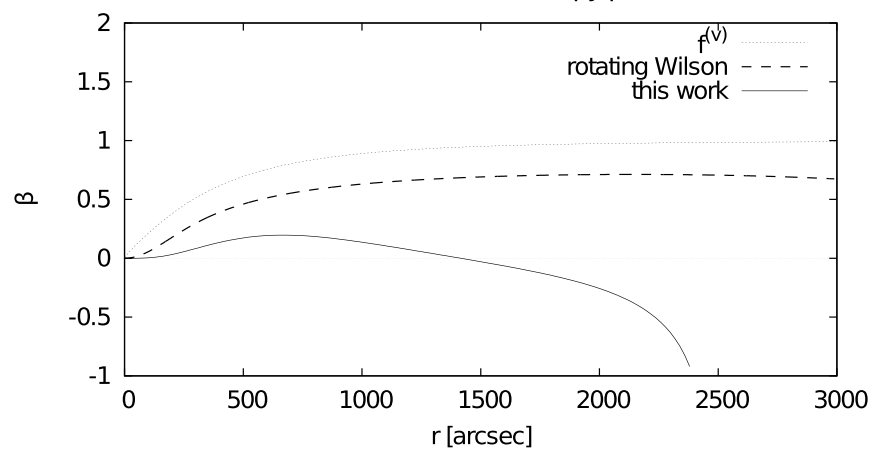

Figure 13. Comparison between our rotating quasi-relaxed model (solid lines), spherical radially biased anisotropic $f^{(v)}$ model (from ZBV12; thin dotted lines), and rotating Wilson (1975) model (from Sollima et al. 2009; dashed lines) for $\omega$ Cen. The top panel represents the projected line-of-sight velocity dispersion profile and the bottom panel represents the intrinsic anisotropy profile defined as $\beta=1-\left(\sigma_{\theta}^{2}+\sigma_{\phi}^{2}\right) / 2 \sigma_{r}^{2}$, evaluated along the equatorial plane. A higher degree of radially biased anisotropy in the central-intermediate region contributes to steepen the central dispersion profile.

based on a rotating Wilson (1975) model; the latter model generates deviations from sphericity in better agreement with the observations in the inner regions, but not in the outer parts (beyond $\approx 1000$ arcsec) where the model is radially anisotropic, whereas $\omega$ Cen is tangentially anisotropic.

We conclude that the structure of $\omega$ Cen is determined by the complex interplay between rotation and anisotropy; significant pressure anisotropy can be naturally present even in its inner regions because this cluster is characterized by long relaxation times.

When applied to the two more relaxed clusters, 47 Tuc and M15, our models perform very well; the systems are quasi-isotropic in their inner regions and internal rotation is able to explain the observed morphology. The most significant discrepancy left is probably that of the core structure of M15 (inside $\approx 10$ arcsec), characterized by a cusp in the surface brightness that is likely to be related to the phenomenon of core collapse (Murphy et al. 2011), which goes beyond the objectives of our equilibrium models. For this cluster, the intermediate and outer regions (from 10 arcsec out to 1000 arcsec) are well fit by our rotating model (Figure 10), at variance with the spherical King model, which severely underestimates the surface brightness (beyond $\approx 300$ arcsec; see Figure 1 in ZBV12).

\subsection{Comparison with Previous Studies}

To our knowledge, an application of nonspherical models to the full set of data available for these clusters, including proper motions, has been made only by van de Ven et al. (2006) for $\omega$ Cen and by van den Bosch et al. (2006) for M15, based on a Schwarzschild-type modeling procedure. Remarkably, the bestfit model for M15 is characterized by a total mass and a mass-tolight ratio fully consistent with our results, that is, $4.4 \times 10^{5} M_{\odot}$ and $1.6 M_{\odot} / L_{\odot}$, respectively. In the case of $\omega$ Cen, we derive a lower value for the total mass and a higher value for the mass-to-light ratio. Here, the discrepancy reflects our estimate of the distance to the object, smaller than distances reported in the literature ${ }^{9}$ (by adopting a distance of $d=4.8 \mathrm{kpc}$, the resulting total mass associated with our rotating model would be $M=2.28 \times 10^{6} M_{\odot}$, whereas for $d=5.2 \mathrm{kpc}$, the total mass would be $M=2.47 \times 10^{6} M_{\odot}$ ).

In addition, only very few studies have been done of nonspherical rotating models constructed under given physical assumptions. To our knowledge, only three families of models based on a distribution function allowing for internal rotation have been explored in significant detail: those by Prendergast \& Tomer (1970), Wilson (1975), and Lupton \& Gunn (1987). The first two were originally designed to describe elliptical galaxies and not GCs. In fact, the closest and most modern paper that we are aware of for which some comparison with the present article could be made, is that by Sollima et al. (2009), although the application presented there is limited to the line-of-sight kinematics (and thus does not consider the star proper-motion data). The comparison was provided in the previous subsection.

Therefore, we are left with the task of comparing the results of the dynamical analysis performed in the present paper with the results obtained from previous studies based on spherical nonrotating models. This comparison is also interesting, because it shows to what extent the determination of the structural parameters is sensitive to the model adopted, or, in other words, to what extent some idealized, relatively simple, commonly used models are likely to introduce systematic errors in probing the structure of GCs. Table 5 summarizes and compares the following derived structural properties: concentration parameter $C$, core radius $R_{\mathrm{c}}$, total mass $M$, and global $V$-band mass-tolight ratio $M / L_{\mathrm{V}}$. In general, the values of the derived structural parameters are consistent with the values derived from other studies. Spherical, nonrotating Wilson models tend to lead to larger truncation radii, as expected.

We note that our rotating models give a good description of the global kinematics and morphology of the three analyzed GCs. As a result, the effects of mass segregation are expected to be modest; in addition, we do not have to invoke the presence of dark matter and we do not find any reason to abandon Newtonian dynamics and to move to the MOdified Newtonian Dynamics (MOND) framework.

\section{3. $V / \sigma$ versus $\varepsilon$}

Finally, we wish to comment on a simple tool commonly used to assess the importance of rotation in determining the global shape of a stellar system, that is, the plot of $V / \sigma$ versus $\varepsilon$ (in the context of elliptical galaxies, see Davies et al. 1983; Emsellem et al. 2011). In Figure 14, the quantity $V / \sigma$ is the ratio of the

\footnotetext{
9 For $\omega$ Cen, the recent investigation by D'Souza \& Rix (2013) assumes a distance of $5.5 \mathrm{kpc}$, much higher than the distance $(4.11 \mathrm{kpc})$ that we determined in the present paper. Based on a discrete kinematic approach, including flattening and rotation, the authors report a value of the total mass of $(4.05 \pm 0.10) \times 10^{6} M_{\odot}$. By assuming an apparent visual magnitude of $m_{\mathrm{V} \text {,tot }}=3.68 \mathrm{mag}$ (Harris 2010), and by rescaling this value to the distance of $5.5 \mathrm{kpc}$ (to obtain the absolute total luminosity), the corresponding mass-to-light ratio is $M / L_{\mathrm{V}}=4.56 M_{\odot} / L_{\odot}$, significantly larger than usually obtained for this cluster.
} 
Table 5

Comparison of the Structural Parameters from the Best-fit Models of the Present Paper with Those Obtained from Spherical Models in Previous Studies

\begin{tabular}{|c|c|c|c|c|c|}
\hline \multirow[t]{2}{*}{$\overline{\mathrm{GC}}$} & \multirow[t]{2}{*}{ Ref. } & \multirow[t]{2}{*}{ C } & \multirow[t]{2}{*}{$R_{\mathrm{c}}$} & $M$ & $M / L_{\mathrm{V}}$ \\
\hline & & & & \multicolumn{2}{|c|}{$\begin{array}{c}\text { Rescaled to a } \\
\text { Common Distance }\end{array}$} \\
\hline \multirow[t]{4}{*}{$\omega$ Cen } & (0) & $1.27 \pm 0.01$ & $127.8 \pm 1.1$ & $24.71 \pm 0.20$ & $2.26 \pm 0.11$ \\
\hline & (1) & $1.32 \pm 0.01$ & $127.7 \pm 2.4$ & $26.45 \pm 3.32$ & $1.93 \pm 0.24$ \\
\hline & (2) & $1.43 \pm 0.02$ & $164.6 \pm 4.5$ & $24.66 \pm 2.26$ & $2.24_{-0.82}^{+1.04}$ \\
\hline & (3) & $1.31 \pm 0.04$ & $142.2 \pm 8.3$ & & \\
\hline \multirow[t]{4}{*}{47 Tuc } & (0) & $1.87 \pm 0.01$ & $24.6 \pm 0.1$ & $6.76 \pm 0.04$ & $1.56 \pm 0.12$ \\
\hline & (1) & $2.01 \pm 0.00$ & $22.6 \pm 0.2$ & $7.18 \pm 0.41$ & $1.34 \pm 0.08$ \\
\hline & (2) & $2.57 \pm 0.06$ & $32.1 \pm 2.6$ & $10.71 \pm 0.98$ & $1.17_{-0.43}^{+0.52}$ \\
\hline & (3) & $2.07 \pm 0.03$ & $21.6 \pm 1.3$ & $\ldots$ & $\ldots^{-0.43}$ \\
\hline \multirow[t]{4}{*}{ M15 } & (0) & $1.94 \pm 0.02$ & $12.9 \pm 0.2$ & $4.49 \pm 0.07$ & $1.47 \pm 0.05$ \\
\hline & (1) & $1.86 \pm 0.01$ & $7.5 \pm 0.1$ & $3.98 \pm 0.35$ & $1.12 \pm 0.10$ \\
\hline & (2) & $\ldots$ & $\ldots$ & $\ldots$ & $\ldots$ \\
\hline & (3) & $2.29 \pm 0.18$ & $8.4 \pm 1.0$ & $\ldots$ & $\ldots$ \\
\hline
\end{tabular}

Notes. For each cluster we provide the concentration parameter $C=\log \left(r_{\mathrm{tr}} / R_{\mathrm{c}}\right)$, the projected core radius $R_{\mathrm{c}}$ in arcsec, the total mass of the cluster $M$ in units of $10^{5} M_{\odot}$, and the $V$-band mass-to-light ratio $M / L_{V}$ in solar units. The values of $M$ and $M / L_{V}$ have been rescaled to a common distance for each cluster to allow for a comparison of the different models considered $(5.2 \mathrm{kpc}, 4.5 \mathrm{kpc}$, and $10.4 \mathrm{kpc}$, for $\omega$ Cen, 47 Tuc, and M15, respectively).

References. (0) This paper; (1) spherical King models from ZBV12; (2) spherical nonrotating Wilson models from MLvdM05; (3) Harris 2010.

observed maximum of the line-of-sight rotation profile to the central line-of-sight velocity dispersion, as reported in Column 3 of Table 2; the values of the ellipticity $\varepsilon$ are those reported by WS87. We indicate the $(V / \sigma, \varepsilon)$ pairs by filled symbols. The empty symbols show the effect of correcting these values for inclination (the assumed inclinations are those reported in Table 1), as if the GCs were viewed "edge-on" $\left(i=90^{\circ}\right)$, following the procedure described in Cappellari et al. (2007). In the figure, the dashed line indicates the relation expected for isotropic oblate rigid rotators viewed "edge-on," whereas the thin dotted lines indicate oblate rotators (viewed "edge on") with different global anisotropy parameters $\delta$ (Binney 2005; Cappellari et al. 2007). Even though rotation and pressure anisotropy vary significantly with radius, ${ }^{10}$ according to this diagram, the flattening observed in the three GCs could be argued to have originated due to the presence of internal rotation. The deviations from the line of isotropic rotators may be interpreted as due to the combined effects of inclination, differential rotation, and pressure anisotropy. The physically simple self-consistent models that we have tested in this paper give insight into how the various physical ingredients may combine their roles into the observed data. The cluster for which the deviation is most significant is $\omega$ Cen, confirming its anomalous behavior (which we have argued to be the result of its only partially relaxed state). This result is even more striking if we refer to the points corrected for inclination.

\section{SUMMARY AND CONCLUSIONS}

In the present paper, we have applied a family of selfconsistent global dynamical models, recently constructed with the purpose of describing differentially rotating star clusters in

\footnotetext{
10 In fact, a diagram has been proposed for a suitably defined luminosity-weighted average of $V / \sigma$; note that within the analytical family of models that we are using, this and other differential indicators of the role of rotation can be constructed in a straightforward way.
}

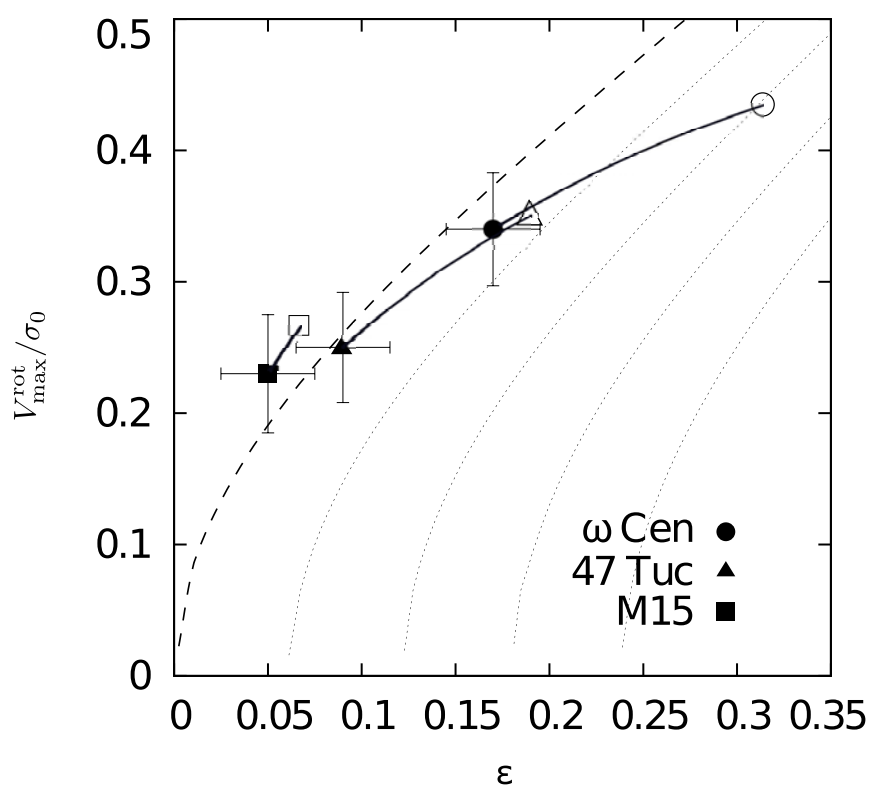

Figure 14. $V / \sigma$ vs. ellipticity $\varepsilon$ for $\omega$ Cen, 47 Tuc, and M15. The filled symbols denote the pairs $(V / \sigma, \varepsilon)$, in which the ellipticity values are determined by WS87. The empty symbols, connected by a segment to the associated filled symbols, indicate the pairs $(V / \sigma, \varepsilon)$ corrected for inclination. The dashed line indicates the relation for isotropic oblate rotators viewed "edge-on," whereas the thin dotted lines indicate oblate rotators viewed "edge-on" with different global anisotropy parameters $\delta$ (from left to right, $\delta=0.05,0.1,0.15$, and 0.20 ). See the text for a more complete description.

a quasi-relaxed state, to three Galactic GCs, namely, $\omega$ Cen, 47 Tuc, and M15, that exhibit evidence for flattening and rotation. For these clusters, an extremely rich set of data is available, particularly on their three-dimensional kinematics.

With respect to the traditional modeling of GCs, generally limited to a study of surface brightness profiles (but see the effort made by ZBV12), we have given here highest priority to the interpretation of the available kinematical data. This is a particularly challenging test for the models. In turn, the success of the models for the two clusters known to be in a sufficiently well-relaxed state allows us to measure their internal structural parameters accurately and reliably, well beyond the reach of simpler and more idealized models.

The modeling procedure is based on three steps. (1) We identify the relevant range of the model parameters from the characteristics of the observed differential rotation. (2) We set the relevant physical scales by means of a standard fitting procedure of the photometric profile and the line-of-sight kinematic profiles. (3) We use the models thus fully identified to make definite, quantitative predictions on several other observational data not used in the first two steps, namely, the anisotropy profile $\sigma_{t} / \sigma_{R}$, the proper-motion mean-velocity profiles $V_{t}$ and $V_{R}$, the ellipticity profile $\varepsilon$, and a map of the relevant projected isodensity contours. The quality and reliability of the adopted family of models is best assessed in this last predictive step, where we do not have free model parameters available anymore. Finally, by combining the gathered information of the proper-motion velocity dispersion with that of the line-of-sight velocity profiles, we obtain a dynamical estimate of the distance to the cluster.

An application of nonspherical models to the full set of data available for these clusters, including proper motions, has been made only by van de Ven et al. (2006) for $\omega$ Cen and by van den Bosch et al. (2006) for M15, based on a Schwarzschild orbitbased modeling procedure. Both modeling techniques assume 
axisymmetry and allow for internal rotation and anisotropy in velocity space. Our physically simple models are based on a distribution function defined in terms of two integrals of the motion and applied under the hypothesis of a constant mass-to-light ratio, whereas the orbit-based models include the possibility of a varying mass-to-light ratio and the presence of a third integral of the motion. Therefore, the latter approach allows for a more general investigation with a relatively free and more complex structure of the pressure anisotropy profile. Despite these differences, we obtain consistent results for the total mass estimates, for the global mass-to-light ratios, and, in particular, for the presence of tangential anisotropy in the outer parts of $\omega$ Cen. To some extent, the descriptive orbitbased modeling and the predictive distribution-function-based modeling are complementary: the fact that they lead to similar results is highly nontrivial and strengthens the conclusions that are obtained.

An important merit of using a physically based family of models is to make predictions. Indeed, we offer a hopefully general physical interpretation of the observed features (in particular, in relation to the interplay between rotation and anisotropy in determining the internal structure of the stellar systems) that might be tested soon on other clusters. In addition, for the three clusters considered in this paper, we make specific predictions about the structural properties in their outer parts that may be tested by future observations (e.g., anisotropy profile, rotation profile, and isodensity contours).

The main results obtained in this paper are the following.

1. For the three most studied GCs, we have illustrated how such a detailed modeling procedure can be implemented to test the adequacy of a physically justified, global, selfconsistent family of models to interpret all the available photometric and kinematic data, including a rich set of proper motions. One important technical problem, the inclination and projection of self-consistent models constructed from a nontrivial distribution function, has been resolved by the use of a suitable discrete realization in terms of a large number of simulated particles (see Section 3.1).

2. For the well-relaxed cluster 47 Tuc, the model that we have identified provides a very good interpretation of the photometric and kinematic data. In particular, the rotation profile is well matched throughout the entire extent of the cluster, showing clearly the position of the maximum rotation velocity, the characteristic rigid rotation behavior in the central region, and the relatively sharp decline in the outer parts. In addition, the proper-motion rotation measured by Anderson \& King (2003) is well consistent with the value predicted by our model at the relevant radial positions. The identification of the model comes out naturally and leads to a specific prediction of the ellipticity profile that is in excellent agreement with the observations.

3 . For the relaxed cluster M15, we provide a global model in good agreement with the data; in particular, the line-ofsight rotation profile in the central regions, characterized by a solid-body behavior, is well accounted for by the model. The possible presence of a fast-rotating core on a small radial scale (where the observed photometric profile shows evidence of a post-core-collapse phase) does not appear to influence the quality of our global description.

4. The model selected for $\omega$ Cen is unable to reproduce the steep central gradient in the line-of-sight velocity dispersion profile; in addition, the predicted ellipticity profile is somewhat offset with respect to the observed profile. We showed how these features are likely to reflect the condition of only partial relaxation of the cluster, as confirmed by the measured high radial anisotropy. Still, somewhat surprisingly, our model provides a satisfactory global interpretation of the complex three-dimensional kinematics available for this object. In particular, the overall behavior of the anisotropy profile is successfully described, including the presence of tangential anisotropy in the outer parts of the system.

5. The results of this study confirm that internal rotation is indeed responsible for the observed flattening for at least two of the three clusters (47 Tuc and M15). For $\omega$ Cen there is no doubt that rotation is important; still, the discrepancy between the predicted and observed ellipticity profiles suggests that pressure anisotropy cooperates in determining its observed morphology.

6. We have determined new dynamical estimates of the distances to the stellar systems under consideration. Before, this kind of analysis had been performed in only a few cases (in particular, see van de Ven et al. 2006; Anderson \& van der Marel 2010). It appears that the distance estimates based on dynamical models are generally lower than those derived from photometric methods, such as the analyses of variable stars (e.g., see Bono et al. 2008) and from other stellar indicators.

Further interesting insights may come from the study of rotation in different environments, such as in low-mass stellar systems in the Magellanic Clouds, where GCs are known to be younger and flatter than the Galactic clusters. In particular, strong differential rotation may be a critical ingredient in determining the structure and internal dynamics of the class of the so-called ring clusters (see Hill \& Zaritsky 2006; Werchan \& Zaritsky 2011), which are characterized by an off-centered peak density profile. The presence of internal rotation may also play an important role in the dynamics of low-mass stellar systems in the transition region between classical star clusters and dwarf galaxies (e.g., see the recent spectroscopic study of the rotating ultracompact dwarf performed by Frank et al. 2011).

We showed that rotation plays an important role in determining the structure of the three clusters considered in this paper, but that morphological information (as exemplified by the ellipticity profile of $\omega \mathrm{Cen}$ ) can be decisive in assessing the quality of a model. It remains to be ascertained how frequently rotation is the key dynamical factor and which GCs owe their shape instead mainly to external tides or simply to pressure anisotropy. New observational efforts to study the morphology of low-mass stellar systems (in particular, devoted to the measurement of ellipticity profiles, isophotal contours, and quadrupole moments) are thus highly desired.

The inclination angle of the objects (assumed here to be axisymmetric) is a key ingredient in modeling the data. This quantity is difficult to measure. Here, for the three clusters, we have adopted the inclination values reported in the literature. Based on the experience developed in this paper, we have devised a new method to determine simultaneously distance and inclination angle for a given axisymmetric stellar system, by means of the combined use of proper motions and line-ofsight velocities under the only assumption that the underlying distribution function depends on the two classical integrals of the motion $f=f\left(E, J_{z}\right)$. We plan to present this result soon, in a separate paper.

Finally, we wish to reiterate (see also ZBV12) that many key dynamical issues (such as a reliable estimate of the dark matter 
content, the search of dynamical signatures of a possible central IMBH, and the evaluation of the effects of mass segregation) can be addressed exclusively by considering appropriate kinematical data in detail (for a recent study with a generally similar approach, but limited to the study of line-of-sight kinematic data, see Sollima et al. 2012). We thus hope that the detailed study presented in this paper may mark the beginning of fruitful developments in the study of the dynamics of GCs and other small-mass stellar systems, beyond the application of exceedingly idealized spherical, nonrotating models so far used almost universally.

We are grateful to A. Sollima for providing us with the kinematic profiles of his best-fit Wilson rotating model for $\omega$ Cen. We thank M. Bellazzini, M. Gieles, D.C. Heggie, G. van de Ven, F. van Leeuwen, and E. Vesperini for useful comments and conversations. Finally, we wish to thank the referee for many constructive remarks that have helped improve the quality of the paper. This work was partially supported by the Italian MIUR.

\section{APPENDIX A}

\section{THE CONSTRUCTION OF THE KINEMATIC DATA SETS USED IN THE PAPER}

For $\omega$ Cen, two different data sets of line-of-sight velocities are considered: 1589 line-of-sight velocities from Reijns et al. (2006) and 649 line-of-sight velocities from Pancino et al. (2007) for the central part of the cluster. After identifying the stars in common between the two samples, the one with the lower associated error is kept. The final combined data set is composed of $N_{\text {los }}=1868$ data, reaching a radial extent of approximately half the truncation radius, with an average error of $1.98 \mathrm{~km} \mathrm{~s}^{-1}$. The proper-motion data available are the ones from van Leeuwen et al. (2000), with a total of 9847 ground-based measurements, and the ones from Anderson \& van der Marel (2010), with a total of 72,970 HST measurements. We treat the two data samples as distinct. In the van Leeuwen et al. (2000) data set, each star is provided with a membership probability and is classified according to the disturbance of the image due to neighboring stars on a scale from 0 to 4 (i.e., from non-disturbed to highly disturbed stars). We decided to select a subsample composed of stars with a membership probability higher than $68 \%$, belonging to class 0 , and with error measurements lower than 0.25 mas yr$^{-1}$ (for a similar selection, see van de Ven et al. 2006). We obtain a sample composed of $N_{\mathrm{pm}}=2740$ proper motions, with a radial extent of approximately half the truncation radius and an average error of $0.16 \mathrm{mas} \mathrm{yr}^{-1}$ (corresponding to $3.89 \mathrm{~km} \mathrm{~s}^{-1}$ for an assumed distance of $5.2 \mathrm{kpc}$ ). The data set from Anderson \& van der Marel (2010) is composed of two fields: a central field within $R \lesssim R_{\mathrm{c}}$ and a field positioned along the major axis between $0.7 R_{\mathrm{c}} \lesssim R \lesssim 2.5 R_{\mathrm{c}}$. The average error of the data is $0.078 \mathrm{mas} \mathrm{yr}^{-1}$ (corresponding to $1.92 \mathrm{~km} \mathrm{~s}^{-1}$ for an assumed distance of $5.2 \mathrm{kpc})$.

For 47 Tuc, the line-of-sight velocities data set results from two data sets combined by following the procedure described in ZBV12: 499 line-of-sight velocities from Gebhardt et al. (1995) for the inner region $(R<100 \mathrm{arcsec})$ and 1977 lineof-sight velocities from Lane et al. (2011) for the outer parts $(R>100 \mathrm{arcsec})$. As noted in Lane et al. (2011), the latter data set shows a mean velocity of $-16.85 \mathrm{~km} \mathrm{~s}^{-1}$, which differs significantly from the value obtained from the former data set, $-18.34 \mathrm{~km} \mathrm{~s}^{-1}$; this is likely to be due to a systematic uncertainty between the zero point of the two velocity systems. To correct for this offset, we have subtracted from each data set the corresponding measured mean velocities. The final line-ofsight velocities sample is composed of $N_{\text {los }}=2476$ velocities covering the entire extent of the cluster with an average error of $2.29 \mathrm{~km} \mathrm{~s}^{-1}$. The proper motions are taken from McLaughlin et al. (2006): $N_{\mathrm{pm}}=12,974 \mathrm{HST}$ proper motions selected on the basis of the star magnitude $(V<20)$ and quality (i.e., we consider data with probability $\left.P\left(\chi^{2}\right)>0.001\right)$; unfortunately, the data cover only the central region out to $\approx 100$ arcsec (approximately 4 core radii). The measurements have an average error of 0.27 mas $\mathrm{yr}^{-1}$ (corresponding to $5.76 \mathrm{~km} \mathrm{~s}^{-1}$ at a distance of $4.5 \mathrm{kpc}$ ).

For M15, we used a single data set composed of $N_{\text {los }}=1777$ line-of-sight velocities from Gebhardt et al. (2000); this sample is centrally concentrated, with $\approx 80 \%$ of the stars being inside $10 R_{c}$. The average error is $3.79 \mathrm{~km} \mathrm{~s}^{-1}$. In addition, we used the sample of $N_{\mathrm{pm}}=703 H S T$ proper motions in the central region of the cluster $\left(R<2 R_{c}\right)$, as reported by McNamara et al.

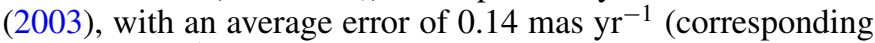
to $6.79 \mathrm{~km} \mathrm{~s}^{-1}$ at a distance of $10.2 \mathrm{kpc}$ ).

We recall that the procedure used to obtain the propermotion data sets described above will not reveal any solidbody rotation in the plane of the sky, as well as any systematic motions of contraction or expansion (e.g., see Vasilevskis et al. 1979; McLaughlin et al. 2006; Anderson \& van der Marel 2010) because the proper-motion measurements are relative measurements (no absolute reference frame is available for measuring the star displacements at different epochs). van de Ven et al. (2006) show how to compensate for the missed solid-body component under the assumption of axisymmetry in the proper-motion sample of van Leeuwen et al. (2000) by combining line-of-sight velocities and proper motions. We apply the related correction to the $\omega$ Cen proper-motion sample of van Leeuwen et al. (2000), while we do not correct the one from Anderson \& van der Marel (2010). For 47 Tuc and M15, given the fact that the data sets are centrally concentrated, we argue that in the very central regions of the clusters, the amount of solid-body rotation associated with this effect is negligible and therefore we do not apply any correction (see van den Bosch et al. 2006, who first noted that the result of the correction for M15 is below the measurement errors and therefore can be ignored). Therefore, for the last two clusters, no sign of rotation in the plane of the sky is expected from the proper-motion data sets considered above; however, rotation in the plane of the sky has been clearly detected for 47 Tuc by Anderson \& King (2003), using as an absolute reference the background stars of the Small Magellanic Cloud.

Finally, an additional correction is applied to the $\omega$ Cen and 47 Tuc data, to correct for the apparent rotation resulting from their large angular extent and their global orbital motion in the Galaxy; to this purpose, we followed closely the procedure described by van de Ven et al. (2006).

\section{APPENDIX B}

\section{TESTS OF THE DETERMINATION OF THE ROTATION POSITION ANGLE AND AMPLITUDE}

To check whether the rotation patterns of the GCs under study show radial variation of the P.A. and the rotation amplitude, we repeated the procedure outlined in Section 2.1.1 on subsamples of data with $R<R_{\max }$, for decreasing values of $R_{\max }$. Table 6 lists the results of the P.A.s and rotation amplitudes for given 
Table 6

Internal Rotation: Position Angle of the Rotation Axis and Rotation Amplitude for Disks of Different Radii

\begin{tabular}{|c|c|c|c|}
\hline \multicolumn{4}{|c|}{$\omega$ Cen } \\
\hline $\begin{array}{l}R_{\max } \\
\text { (1) }\end{array}$ & $\begin{array}{c}A \\
(2)\end{array}$ & $\begin{array}{l}\text { P.A. } \\
\text { (3) }\end{array}$ & $\begin{array}{l}N \\
(4)\end{array}$ \\
\hline all & 6.79 & 12 & 1868 \\
\hline $10 R_{c}$ & 6.91 & 12 & 1827 \\
\hline $8 R_{c}$ & 7.09 & 10 & 1737 \\
\hline $6 R_{c}$ & 7.73 & 7 & 1481 \\
\hline $4 R_{c}$ & 7.58 & 11 & 1026 \\
\hline $2 R_{c}$ & 6.95 & 22 & 398 \\
\hline $1 R_{C}$ & 3.97 & 57 & 91 \\
\hline $0.9 R_{c}$ & 1.25 & -4 & 73 \\
\hline $0.7 R_{c}$ & 1.98 & 23 & 42 \\
\hline $0.6 R_{c}$ & 7.04 & 10 & 27 \\
\hline $0.5 R_{c}$ & 13.93 & -8 & 19 \\
\hline
\end{tabular}

\begin{tabular}{|c|c|c|c|}
\hline $\begin{array}{l}R_{\max } \\
(1)\end{array}$ & $\begin{array}{c}A \\
(2)\end{array}$ & $\begin{array}{c}\text { P.A. } \\
\text { (3) }\end{array}$ & $\begin{array}{l}N \\
(4)\end{array}$ \\
\hline all & 4.00 & 136 & 2476 \\
\hline $80 R_{c}$ & 4.11 & 136 & 2414 \\
\hline $40 R_{c}$ & 4.41 & 137 & 2058 \\
\hline $20 R_{c}$ & 4.53 & 136 & 1358 \\
\hline $10 R_{c}$ & 3.32 & 139 & 800 \\
\hline $5 R_{c}$ & 2.24 & 164 & 526 \\
\hline $2 R_{c}$ & 2.64 & 180 & 388 \\
\hline $1 R_{c}$ & 4.07 & 199 & 114 \\
\hline $0.8 R_{c}$ & 4.05 & 171 & 78 \\
\hline $0.7 R_{c}$ & 5.99 & 167 & 61 \\
\hline $0.6 R_{c}$ & 4.78 & 206 & 39 \\
\hline \multicolumn{4}{|c|}{ M15 } \\
\hline$R_{\max }$ & $A$ & P.A. & $N$ \\
\hline (1) & (2) & (3) & (4) \\
\hline all & 2.84 & 106 & 1777 \\
\hline $30 R_{c}$ & 2.89 & 106 & 1671 \\
\hline $10 R_{c}$ & 2.93 & 102 & 1467 \\
\hline $8 R_{c}$ & 3.00 & 99 & 1293 \\
\hline $5 R_{c}$ & 1.94 & 118 & 916 \\
\hline $4 R_{c}$ & 1.43 & 140 & 724 \\
\hline $2 R_{c}$ & 2.14 & 147 & 319 \\
\hline $1 R_{c}$ & 1.19 & 253 & 128 \\
\hline $0.6 R_{c}$ & 4.68 & 272 & 65 \\
\hline $0.5 R_{c}$ & 6.95 & 253 & 52 \\
\hline $0.4 R_{c}$ & 13.00 & 261 & 31 \\
\hline
\end{tabular}

Notes. For each cluster we report the value of the position angle of the rotation axis P.A. measured in degrees east of north (Column 3) and the rotation amplitude $A$ in $\mathrm{km} \mathrm{s}^{-1}$ (Column 2) obtained from a fit of a sine function when considering $N$ data (Column 4 ) inside $R_{\max }$ (Column 1). For each cluster, the first row corresponds to the results illustrated in Figure 1.

values of $R_{\max }$. To assess whether the number of data available for the different cases is sufficiently large to obtain a significant measure of the P.A.s and the rotation amplitudes, we tested the method used on simulated data drawn from a rotating model of the family introduced in Section 3. We found that the estimates of the P.A.s obtained from samples of data with $N \lesssim 100$ have a typical uncertainty (associated with a $68 \%$ confidence level) greater than $\pm 25^{\circ}$. We conclude that no significant P.A. variation is present in 47 Tuc and $\omega$ Cen, whereas for M15 a twisting is detected from $260^{\circ}$ in the innermost region (on the scale of the core radius) to $106^{\circ}$ in the outer parts (thus confirming the result found by Gebhardt et al. 2000).
Moreover, we found that the rotation amplitude $A$ changes across the clusters. In general, it reaches a maximum at intermediate values of $R_{\max }$. This can be taken as an indication of differential rotation (as illustrated by the shape of the rotation profiles, see Figures 2, 7, and 10). Interestingly, all three clusters show a sharp increase of the rotation amplitude in the very central regions. This feature may be interpreted as a signature of a complex rotation pattern, characterized by a rapidly spinning core, as reported by van de Ven et al. (2006) and van den Bosch et al. (2006), ascribed to a disk-like rotating component in $\omega$ Cen and a decoupled rotating core in M15. The last rows in Table 6 show that $\omega$ Cen reaches an amplitude of $A=13.93 \mathrm{~km} \mathrm{~s}^{-1}$ for $R<0.5 R_{c}, 47$ Tuc has $A=4.78 \mathrm{~km} \mathrm{~s}^{-1}$ for $R<0.6 R_{c}$, and M15 has $A=13.00 \mathrm{~km} \mathrm{~s}^{-1}$ for $R<0.4 R_{c}$.

To test the significance of the detected central rotation, we performed a Monte Carlo simulation. We draw from a nonrotating model, characterized by a realistic value of the central concentration, a simulated data set with an equal number of data and similar spatial distribution with respect to the real case (see last row of Table 6). We then computed for each cluster $N=1000$ random realizations of this synthetic data set and we applied to them the procedure to calculate the rotation amplitude $A$, as described above in Section 2.1.1. Finally, from the distribution of the derived rotation amplitudes, we calculated the probability of finding a value of $A$ higher than the one derived from the real data. We found that the probability of measuring by chance rotation amplitudes as high as the ones determined when no rotation is present is $7 \%, 32 \%$, and $\lesssim 1 \%$ for $\omega$ Cen, 47 Tuc, and M15, respectively. We thus conclude that the central increase measured in 47 Tuc is not statistically significant, whereas the increase can be taken as a sign of genuine high rotation in the central regions of M15. The presence of high rotation in the core of $\omega$ Cen is less clearly defined.

\section{REFERENCES}

An, J. H., \& Evans, N. W. 2006, AJ, 131, 782

Anderson, J., \& King, I. R. 2003, AJ, 126, 772

Anderson, J., \& van der Marel, R. P. 2010, ApJ, 710, 1032

Baumgardt, H., \& Makino, J. 2003, MNRAS, 340, 227

Baumgardt, H., Makino, J., \& Hut, P. 2005, ApJ, 620, 238

Bellazzini, M., Bragaglia, A., Carretta, E., et al. 2012, A\&A, 538, A18

Bellini, A., Piotto, G., Bedin, L. R., et al. 2009, A\&A, 493, 959

Binney, J. 2005, MNRAS, 363, 937

Bono, G., Stetson, P. B., Sanna, N., et al. 2008, ApJL, 686, L87

Cappellari, M., Emsellem, E., Bacon, R., et al. 2007, MNRAS, 379, 418

Chen, C. W., \& Chen, W. P. 2010, ApJ, 721, 1790 (CC10)

Côté, P., Welch, D. L., Fischer, P., \& Gebhardt, K. 1995, ApJ, 454, 788

Davies, R. L., Efstathiou, G., Fall, S. M., Illingworth, G., \& Schechter, P. L. 1983, ApJ, 266, 41

D'Souza, R., \& Rix, H.-W. 2013, MNRAS, 429, 1887

Einsel, C., \& Spurzem, R. 1999, MNRAS, 302, 81

Emsellem, E., Cappellari, M., Krajnović, D., et al. 2011, MNRAS, 414, 888

Fall, S. M., \& Frenk, C. S. 1985, in IAU Symp. 113, Dynamics of Star Clusters, ed. J. Goodman \& P. Hut (Dordrecht: Reidel), 285

Fiestas, J., Spurzem, R., \& Kim, E. 2006, MNRAS, 373, 677

Frank, M. J., Hilker, M., Mieske, S., et al. 2011, MNRAS, 414, L70

Gebhardt, K., Pryor, C., O'Connell, R. D., Williams, T. B., \& Hesser, J. E. 2000, AJ, 119, 1268

Gebhardt, K., Pryor, C., Williams, T. B., \& Hesser, J. E. 1995, AJ, 110, 1699

Gerssen, J., van der Marel, R. P., Gebhardt, K., et al. 2002, AJ, 124, 3270

Geyer, E. H., Nelles, B., \& Hopp, U. 1983, A\&A, 125, 359

Harris, W. E. 2010, arXiv:1012.3224

Heggie, D., \& Hut, P. 2003, The Gravitational Million-Body Problem: A Multidisciplinary Approach to Star Cluster Dynamics (Cambridge: Cambridge Univ. Press)

Hill, A., \& Zaritsky, D. 2006, AJ, 131, 414 
Hong, J., Kim, E., Lee, H. M., \& Spurzem, R. 2013, MNRAS, 430, 2960 Hurley, J. R., \& Shara, M. M. 2012, MNRAS, 425, 2872

Kim, E., Einsel, C., Lee, H. M., Spurzem, R., \& Lee, M. G. 2002, MNRAS, 334,310

Kim, E., Yoon, I., Lee, H. M., \& Spurzem, R. 2008, MNRAS, 383, 2

King, I. 1961, AJ, 66, 68

King, I. R. 1966, AJ, 71, 64

Kontizas, E., Kontizas, M., Sedmak, G., \& Smareglia, R. 1989, AJ, 98, 590

Küpper, A. H. W., Kroupa, P., Baumgardt, H., \& Heggie, D. C. 2010, MNRAS, 407,2241

Lane, R. R., Kiss, L. L., Lewis, G. F., et al. 2011, A\&A, 530, A31

Lane, R. R., Küpper, A. H. W., \& Heggie, D. C. 2012, MNRAS, 423, 2845

Lanzoni, B., Mucciarelli, A., Origlia, L., et al. 2013, ApJ, 769, 107

Lupton, R. H., \& Gunn, J. E. 1987, AJ, 93, 1106

Lupton, R. H., Gunn, J. E., \& Griffin, R. F. 1987, AJ, 93, 1114

Lützgendorf, N., Kissler-Patig, M., Noyola, E., et al. 2011, A\&A, 533, A36

McLaughlin, D. E., Anderson, J., Meylan, G., et al. 2006, ApJS, 166, 249

McLaughlin, D. E., \& van der Marel, R. P. 2005, ApJS, 161, 304 (MLvdM05)

McNamara, B. J., Harrison, T. E., \& Anderson, J. 2003, ApJ, 595, 187

McNamara, B. J., Harrison, T. E., \& Baumgardt, H. 2004, ApJ, 602, 264

Merritt, D., Meylan, G., \& Mayor, M. 1997, AJ, 114, 1074

Meylan, G., \& Heggie, D. C. 1997, A\&ARv, 8, 1

Meylan, G., \& Mayor, M. 1986, A\&A, 166, 122

Murphy, B. W., Cohn, H. N., \& Lugger, P. M. 2011, ApJ, 732, 67

Noyola, E., \& Gebhardt, K. 2006, AJ, 132, 447

Noyola, E., Gebhardt, K., \& Bergmann, M. 2008, ApJ, 676, 1008

Pancino, E., Galfo, A., Ferraro, F. R., \& Bellazzini, M. 2007, ApJL, 661, L155

Peterson, R. C., \& Cudworth, K. M. 1994, ApJ, 420, 612

Prendergast, K. H., \& Tomer, E. 1970, AJ, 75, 674
Pryor, C., \& Meylan, G. 1993, in ASP Conf. Ser., Vol. 50, Structure and Dynamics of Globular Clusters, ed. S. G. Djorgovski \& G. Meylan (San Francisco, CA: ASP), 357

Reijns, R. A., Seitzer, P., Arnold, R., et al. 2006, A\&A, 445, 503

Sariya, D. P., Yadav, R. K. S., \& Bellini, A. 2012, A\&A, 543, A87

Sollima, A., Bellazzini, M., \& Lee, J.-W. 2012, ApJ, 755, 156

Sollima, A., Bellazzini, M., Smart, R. L., et al. 2009, MNRAS, 396, 2183

Takahashi, K., \& Lee, H. M. 2000, MNRAS, 316, 671

Trager, S. C., King, I. R., \& Djorgovski, S. 1995, AJ, 109, 218

van de Ven, G., van den Bosch, R. C. E., Verolme, E. K., \& de Zeeuw, P. T. 2006, A\&A, 445, 513

van den Bergh, S. 2008, AJ, 135, 1731

van den Bosch, R., de Zeeuw, T., Gebhardt, K., Noyola, E., \& van de Ven, G. 2006, ApJ, 641, 852

van der Marel, R. P., \& Anderson, J. 2010, ApJ, 710, 1063

van Leeuwen, F., \& Le Poole, R. S. 2002, in ASP Conf. Ser. 265, Omega Centauri, A Unique Window into Astrophysics, ed. F. van Leeuwen \& J. D. Hughes (San Francisco, CA: ASP), 41

van Leeuwen, F., Le Poole, R. S., Reijns, R. A., Freeman, K. C., \& de Zeeuw, P. T. 2000, A\&A, 360, 472

Varri, A. L., \& Bertin, G. 2012, A\&A, 540, A94 (VB12)

Vasilevskis, S., van Leeuwen, F., Nicholson, W., \& Murray, C. A. 1979, A\&AS, 37,333

Werchan, F., \& Zaritsky, D. 2011, AJ, 142, 48

White, R. E., \& Shawl, S. J. 1987, ApJ, 317, 246 (WS87)

Wilson, C. P. 1975, AJ, 80, 175

Woodley, K. A., Goldsbury, R., Kalirai, J. S., et al. 2012, AJ, 143, 50

Zloczewski, K., Kaluzny, J., Rozyczka, M., Krzeminski, W., \& Mazur, B. 2012, AcA, 62, 357

Zocchi, A., Bertin, G., \& Varri, A. L. 2012, A\&A, 539, A65 (ZBV12) 